\title{
Asymptotic Analysis of Gaussian Integrals, II : Manifold of Minimum Points
}

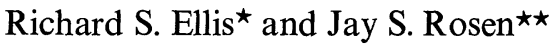 \\ Department of Mathematics and Statistics, University of Massachusetts, Amherst, MA 01003 USA
}

\begin{abstract}
This paper derives the asymptotic expansions of a wide class of Gaussian function space integrals under the assumption that the minimum points of the action form a nondegenerate manifold. Such integrals play an important role in recent physics. This paper also proves limit theorems for related probability measures, analogous to the classical law of large numbers and central limit theorem.
\end{abstract}

\section{Introduction}

In the last few years, theoretical physicists have developed beautiful new ideas for the asymptotic analysis of Gaussian function space integrals [Coleman; Sect. 2], [Wiegel]. In this analysis one is confronted by the "zero mode problem". The object of this paper is to provide the mathematical framework for handling this problem. In particular, we present the complete asymptotic expansions of a class of Gaussian integrals on a Hilbert space, for which the minimum points of the action form a nondegenerate manifold. In addition, we prove limit theorems for related probability measures, analogous to the classical law of large numbers and central limit theorem.

To describe our problem, let $P_{A}$ denote a mean zero Gaussian probability measure with covariance operator $A$ on a real separable Hilbert space $\mathscr{H}$. We write the inner product of $\mathscr{H}$ as $\langle-,-\rangle$. We wish to describe the asymptotics of

$$
J_{n} \doteq \int \psi(Y / \sqrt{n}) \exp (-n F(Y / \sqrt{n})) d P_{A}(Y), \quad \text { as } n \rightarrow \infty .
$$

For simplicity we assume that $\psi, F$ are smooth functionals (smooth will always mean $C^{\infty}$ ), with $\psi$ bounded and $F$ satisfying

$$
F(Y) \geqq-b\|Y\|^{2}-c \text { for some } 0 \leqq b<1 /(2\|A\|), \quad c \geqq 0 .
$$

\footnotetext{
* Alfred P. Sloan Research Fellow. Research supported in part by NSF Grant MCS-80-02149

$\star \star$ Research supported in part by NSF Grant MCS-80-02140
} 
These conditions assure that the integral in (1.1) exists. The covariance operator $A$ is trace class. We also assume that $A$ is strictly positive. In Sect. 6, we extend our results to the Banach space $C[0,1]$, which is important in applications.

Formally, $J_{n}$ can be written as

$$
\begin{aligned}
& \int \psi(Y / \sqrt{n}) \exp \left(-n F(Y / \sqrt{n})-\left\langle A^{-1} Y, Y\right\rangle / 2\right) d Y \\
& \quad=\int \psi(Y / \sqrt{n}) \exp (-n G(Y / \sqrt{n})) d Y,
\end{aligned}
$$

where $G(Y) \doteq\left\langle A^{-1} Y, Y\right\rangle / 2+F(Y)$ is called the (Euclidean) action. In (1.3) $d Y$ is the purely formal translation invariant measure on $\mathscr{H}$. Nevertheless, (1.3) will serve as a useful guide to our intuition.

Let $x$ be a minimum point for $G$, so that $G(x)=\inf _{\mathscr{H}} G$. This implies that

$$
G^{\prime}(x)=0, \quad G^{\prime \prime}(x) \geqq 0,
$$

where $G^{\prime}(x), G^{\prime \prime}(x)$ are respectively the element of $\mathscr{H}$ and the linear operator on $\mathscr{H}$ defined by the first and second Frechet derivatives of $G$ at $x$. (See Sect. 2 for details.) We can then write, for $Y$ near 0 ,

$$
G(x+Y)=G(x)+(1 / 2)\left\langle G^{\prime \prime}(x) Y, Y\right\rangle+\text { error term. }
$$

If $G^{\prime \prime}(x)>0$, then insertion of (1.5) in (1.3) indicates that we can expand around a new Gaussian measure, with covariance operator $\left(G^{\prime \prime}(x)\right)^{-1}$. This we have carried out in detail in [Ellis-Rosen (1)], which generalizes earlier work of [Schilder] and [Pincus].

In the present paper, we assume that the set of minimum points of $G$ forms a smooth submanifold $M$ of $\mathscr{H}$. In this case, differentiating the first equation in (1.4) shows that for any $x \in M$,

$$
G^{\prime \prime}(x) v=0 \text { for all } v \in M_{x},
$$

where $M_{x}$ denotes the tangent space to $M$ at $x$. This degeneracy of $G^{\prime \prime}(x)$ is the "zero mode problem." We note that $M$ is a compact subset of $\mathscr{H}$ (see Lemma 1 (a) in Sect. 2).

The best we can hope for is

$$
G^{\prime \prime}(x)>0 \quad \text { on } N_{x},
$$

where $N_{x} \doteq\left(M_{x}\right)^{\perp}$ is the normal space to $M$ at $x$. We call $M$ nondegenerate if (1.6) holds for all $x \in M$.

Throughout this paper we assume that $M$ is a smooth nondegenerate submanifold of $\mathscr{H}$. However, the ideas presented here together with those of [EllisRosen (1)] enable one to handle many cases of degenerate $M$.

To state our main theorems, we introduce some notation. If $V$ is a subspace of $\mathscr{H}, \pi_{V}$ will denote orthogonal projection onto $V$. If $L$ is a linear operator on $\mathscr{H}$, then $L_{V}, L^{V}$ denote the linear operators on $V$

$$
\begin{aligned}
& L_{V} \doteq \pi_{V} L \pi_{V}, \\
& L_{V} \doteq\left(\pi_{V} L^{-1} \pi_{V}\right)^{-1} .
\end{aligned}
$$

For each specific $L$, we will have to verify that $L_{V}, L^{V}$ are well defined. 
The geometry of $M$ enters through the Weingarten map $W_{x, z}$. For each $x \in M, z \in N_{x}, W_{x, z}$ is the linear transformation of $M_{x}$ defined by

$$
\left\langle W_{x, z} u, v\right\rangle \doteq\left\langle D_{u} \tilde{v}, z\right\rangle, \quad u, v \in M_{x} .
$$

Here $D_{u} \tilde{v}$ is the directional derivative at $x$, in the direction $u$, of any tangent vector field $\tilde{v}$ on $M$ with $\tilde{v}(x)=v$ [Spivak, Vol. IV, p. 49].

Theorem 1 is stated in great generality. A simple special case which should look familiar to physicists is described in (1.18). The main ideas behind Theorem 1 are explained in Sect. 3 before the proof of the theorem.

Theorem 1. Let $M$, the set of minimum points of $G$, be a smooth, nondegenerate submanifold of $\mathscr{H}$. Then $\operatorname{dim}(M)<\infty$ and $e^{n G^{*}} J_{n}$ has the asymptotic expansion (see Explanation below)

$$
\begin{aligned}
e^{n G^{*}} J_{n} \sim n^{\operatorname{dim}(M) / 2} \int_{M}\left\{\int_{N_{x}} \psi(x+z / \sqrt{n}) I_{M}(x, z / \sqrt{n}) .\right. \\
\left.\cdot \exp \left(-n F_{3}(x, z / \sqrt{n})\right) d P_{B(x)}(z)\right\} c(x) d V_{M}(x) \text { as } n \rightarrow \infty,
\end{aligned}
$$

where $G^{*} \doteq \min _{\mathscr{H}} G$,

$$
\begin{gathered}
I_{M}(x, z) \doteq \operatorname{det}\left\{I-W_{x, z}\right\}, \\
F_{3}(x, z) \doteq F(x+z)-F(x)-\left\langle F^{\prime}(x), z\right\rangle-\left\langle F^{\prime \prime}(x) z, z\right\rangle / 2, \\
B(x) \doteq\left(G^{\prime \prime}(x)_{N_{x}}\right)^{-1} \text { is the covariance operator of } P_{B(x)}, \\
c(x) \doteq\left[\operatorname{det}\left(2 \pi A_{M_{x}}\right) \operatorname{det}\left(I+A^{N_{x}}\left(F^{\prime \prime}(x)_{N_{x}}\right)\right)\right]^{-1 / 2},
\end{gathered}
$$

and $d V_{M}$ is the volume-element of $M$ induced by $\mathscr{H}$ [Spivak, Vol. I, p. 423]. The highest order term is $n^{\operatorname{dim}(M) / 2} \int_{M} \psi(x) c(x) d V_{M}(x)$.

Explanation. The smoothness of $\psi, I_{M}(x, \cdot)$, and $F$ implies that for each $x \in M, z \in N_{x}$, the integrand on the right-hand side of (1.9) has an asymptotic expansion of the form

$$
\begin{aligned}
& n^{\operatorname{dim}(M) / 2} \psi(x+z / \sqrt{n}) I_{M}(x, z / \sqrt{n}) \exp \left(-n F_{3}(x, z / \sqrt{n})\right) \\
& \sim n^{\operatorname{dim}(M) / 2} \sum_{j \geqq 0} a_{j}(x, z) n^{-j / 2} \quad \text { as } n \rightarrow \infty,
\end{aligned}
$$

where the $\left\{a_{j}(x, z)\right\}$ are functionals. Then (1.9) means that $\exp \left(n G^{*}\right) J_{n}$ has the asymptotic expansion

$$
e^{n G^{*}} J_{n} \sim n^{\operatorname{dim}(M) / 2} \sum_{j \geqq 0}\left[\int_{M}\left\{\int_{N_{x}} a_{j}(x, z) d P_{B(x)}(z)\right\} c(x) d V_{M}(x)\right] n^{-j / 2} \quad \text { as } n \rightarrow \infty .
$$

For $j$ odd, $a_{j}(x, z)$ turns out to be an odd function of $z$. Since $P_{B(x)}$ is mean zero, only integral powers of $n^{-1}$ appear in the last sum. Thus, (1.9) has the form $n^{\operatorname{dim}(M) / 2} \sum_{j \geqq 0} \Gamma_{j} n^{-j}$, where the $\left\{\Gamma_{j}\right\}$ are functionals.

Remarks. (i) $W_{x, z}$ is related to the second fundamental form of $M$; see [Spivak, 
Vol. IV, p. 49]. We note that if $x(s), s \in R^{m}$, is a local parametrization of $M$, and if $x_{i}(s) \doteq \frac{\partial x}{\partial s_{i}}(s), x_{i j}(s) \doteq \frac{\partial^{2} x(s)}{\partial s_{i} \partial s_{j}}$, then

$$
\left\langle W_{x(s), z} x_{i}(s), x_{j}(s)\right\rangle=\left\langle x_{i j}(s), z\right\rangle .
$$

Thus, if $g(s)$ is the matrix with $g_{k \ell}(s)=\left\langle x_{k}(s), x_{\ell}(s)\right\rangle$, then

$$
W_{x(s), z} x_{i}(s)=\sum_{k} \sum_{j}\left\langle x_{i j}(s), z\right\rangle\left(g(s)^{-1}\right)_{j k} x_{k}(s) .
$$

(ii) In the special case that $M$ is a curve $\left\{f_{s} ; 0 \leqq s \leqq \ell\right\}$ parametrized by arc length, Theorem 1 takes the form

$$
\begin{aligned}
e^{n G^{*}} J_{n} \sim n^{1 / 2} \int_{0}^{\ell}\left\{\int_{\left\{z:\left\langle z, f_{s}^{\prime}\right\rangle=0\right\}} \psi\left(f_{s}+z / \sqrt{n}\right)\left(1-\left\langle\frac{d^{2} f_{s}}{d s^{2}}, \frac{z}{\sqrt{n}}\right\rangle\right) .\right. \\
\left.\cdot \exp \left(-n F_{3}\left(f_{s} ; z / \sqrt{n}\right)\right) d P_{B\left(f_{s}\right)}(z)\right\} d s .
\end{aligned}
$$

We note that $\left\langle\frac{d^{2} f_{s}}{d s^{2}}, z\right\rangle$ is the curvature of $M$ in the direction $z$.

In applications, the manifold $M$ of minimum points often arises because of a continuous group of symmetries. We say that a group $\mathscr{S}$ of unitary transformations of $\mathscr{H}$ is a group of symmetries of $J_{n}$ if for all $s \in \mathscr{S}, Y \in \mathscr{H}$,

$$
\psi(s Y)=\psi(Y), \quad F(s Y)=F(Y), \quad s^{-1} A s=A .
$$

If $\mathscr{S}$ is a group of symmetries of $J_{n}$, then $G$ and consequently $M$ are $\mathscr{S}$-invariant. If in addition

$$
M=\mathscr{S} x_{0} \doteq\left\{s x_{0} \mid s \in \mathscr{S}\right\}
$$

(i.e., if $M$ equals the $\mathscr{S}$-orbit of a single point $x_{0}$ ), then Theorem 1 takes a particularly simple form.

Theorem 2. Assume that $\mathscr{S}$ is a group of symmetries for $J_{n}$ and $M=\mathscr{S} x_{0}$. Then under the assumptions of Theorem 1

$$
\begin{aligned}
& e^{n G^{*}} J_{n} \sim n^{\operatorname{dim}(M) / 2} c\left(x_{0}\right) V(M) \iint_{N_{x_{0}}} \psi\left(x_{0}+z / \sqrt{n}\right) I_{M}\left(x_{0}, z / \sqrt{n}\right) . \\
& \cdot \exp \left(-n F_{3}\left(x_{0} ; z / \sqrt{n}\right)\right) d P_{B\left(x_{0}\right)}(z) \quad \text { as } n \rightarrow \infty
\end{aligned}
$$

where $V(M) \doteq \int_{M} d V_{M}$. The highest order term is $n^{\operatorname{dim}(M) / 2} c\left(x_{0}\right) \psi\left(x_{0}\right) V(M)$.

Remarks. 1. The volume element $d V_{M}$ is the image of Haar measure.

2. Returning to the case of $M$ a curve $\left\{f_{s} ; 0 \leqq s \leqq \ell\right\}$ parametrized by arc length (see (1.14)), Theorem 2 says that in the presence of symmetries

$$
\begin{aligned}
e^{n G^{*}} J_{n} \sim & \sqrt{n} \ell c\left(f_{0}\right) \int_{\left\{z:\left\langle z, f^{\prime} 0\right\rangle=0\right\}} \psi\left(f_{0}+z / \sqrt{n}\right)\left(1-\left\langle\left.\frac{d^{2} f_{s}}{d s^{2}}\right|_{s=0}, \frac{z}{\sqrt{n}}\right\rangle\right) . \\
& \cdot \exp \left(-n F_{3}\left(f_{0} ; z / \sqrt{n}\right)\right) d P_{B\left(f_{0}\right)}(z) .
\end{aligned}
$$


Thus, the highest order term is $n^{1 / 2} \ell c\left(f_{0}\right) \psi\left(f_{0}\right)$ and the next order term involves the curvature of $M$. For comparison, recall that in the case of $M=\left\{f_{0}\right\}$, a nondegenerate critical point, the highest order term is $\bar{c}\left(f_{0}\right) \psi\left(f_{0}\right)$, for some constant $\bar{c}\left(f_{0}\right)$ [Ellis-Rosen (1); Theorem 2.1].

We next describe two limit theorems for probability measures related to $J_{n}$. These limit theorems are analogues of the classical law of large numbers and central limit theorem. Let $Q_{n}$ be the probability measure on $\mathscr{H}$ defined by

$$
Q_{n}(C) \doteq \int_{C} \exp (-n F(Y / n)) d P_{A}(Y / \sqrt{n}) / Z_{n},
$$

where $C$ is a Borel subset of $\mathscr{H}$, and

$$
Z_{n} \doteq \int \exp (-n F(Y / n)) d P_{A}(Y / \sqrt{n})=\int \exp (-n F(Y / \sqrt{n})) d P_{A}(Y)
$$

We have for any integrable $h$

$$
\int h(Y / n) d Q_{n}(Y)=\int h(Y / \sqrt{n}) \exp (-n F(Y / \sqrt{n})) d P_{A}(Y) / Z_{n} .
$$

Theorem 3. Under the hypotheses of Theorem 1, for any bounded, continuous functional $\phi$ on $\mathscr{H}$, we have

$$
\lim _{n \rightarrow \infty} \int \phi(Y / n) d Q_{n}(Y)=\int_{M} \phi(x) c(x) d V_{M}(x) / \int_{M} c(x) d V_{M}(x) .
$$

If $Y_{n}$ denotes the $\mathscr{H}$-valued random vector defined by the identity map $\left(\mathscr{H}, d Q_{n}\right) \rightarrow \mathscr{H}$, then Theorem 3 asserts that $Y_{n} / n$ converges in distribution to the probability measure $d \rho(x) \doteq c(x) d V_{M}(x) / \int c(x) d V_{M}(x)$ concentrated on $M$. We next study the fluctuations of $Y_{n} / n$ around this limiting distribution. The following theorem says that these fluctuations are a $d \rho$-mixture of normal fluctuations in each fiber $N_{x}, x \in M$.

If $\delta>0$ is sufficiently small, we can assign to any $W \in \mathscr{H}$ with $\operatorname{dist}(W, M) \leqq \delta$ a unique closest point $\pi(W)$ in $M$. This follows from the $\varepsilon$-neighborhood theorem [Guilleman-Pollack, p. 69]. If dist $(W, M)>\delta$, then we set $\pi(W)=0$. Since by Theorem $3 Y_{n} / n$ is close to $M, Y_{n} / n-\pi\left(Y_{n} / n\right)$ should be close to zero. To study the fluctuations of $Y_{n} / n$ we will study

$$
\sqrt{n}\left[Y_{n} / n-\pi\left(Y_{n} / n\right)\right]=\frac{Y_{n}-n \pi\left(Y_{n} / n\right)}{\sqrt{n}} .
$$

Theorem 4. Under the hypotheses of Theorem 1, for any bounded continuous functional $\phi$ on $\mathscr{H}$, we have

$$
\lim _{n \rightarrow \infty} \int \phi\left(\frac{Y-n \pi(Y / n)}{\sqrt{n}}\right) d Q_{n}(Y)=\iint_{M}\left\{\int_{N_{x}} \phi(z) d P_{B(x)}(z)\right\} c(x) d V_{M}(x) / \int_{M} c(x) d V_{M}(x) .
$$

In Sect. 2 we present preliminaries which will be needed in our proof of Theorem 1. Sect. 3 contains the proof of Theorem 1. At the beginning of that section, we explain the main ideas behind Theorem 1 . Section 4 contains the proofs of various lemmas used in Sect. 3, and in Sect. 5 we prove Theorems 2, 3, and 4. In Sect. 6, we 
extend our results to $C[0,1]$. An Appendix contains a result on the tightness of certain measures on $C[0,1]$. This result is used in Sect. 6 .

Earlier work on the asymptotics of Gaussian integrals has been done by [Pincus], [Schilder], [Donsker-Varadhan], and [Ellis-Rosen (1)]. Our study of the manifold case is new. We have been informed by $\mathrm{S}$. Breen that for certain cases he has obtained the key change of variables formula discussed in Sect. 3. His methods are quite different from ours.

\section{Preliminaries}

Let $F$ be a $C^{\infty}$ Frechet differentiable functional on the Hilbert space $\mathscr{H}$. Given $\bar{Y}, Y_{1}, \ldots, Y_{j}$ in $\mathscr{H}$, we denote the $j^{\text {th }}$ Frechet derivative of $F$ at $\bar{Y}$ by $D^{j} F(\bar{Y})\left(Y_{1}, \ldots\right.$, $Y_{j}$ ). If $Y_{1}=\ldots=Y_{j}=Y$, we use the notation $D^{j} F(\bar{Y}) Y^{j} \doteq D^{j} F(\bar{Y})\left(Y_{1}, \ldots, Y_{j}\right)$. The first and second Frechet derivatives of $F$ at $\bar{Y}$ define, respectively, an element $F^{\prime}(\bar{Y}) \in \mathscr{H}$ and a bounded symmetric linear operator $F^{\prime \prime}(\bar{Y})$ on $\mathscr{H}$ which satisfy

$$
D^{\prime} F(\bar{Y}) Y_{1}=\left\langle F^{\prime}(\bar{Y}), Y_{1}\right\rangle, \quad D^{2} F(\bar{Y})\left(Y_{1}, Y_{2}\right)=\left\langle F^{\prime \prime}(\bar{Y}) Y_{1}, Y_{2}\right\rangle .
$$

Let $A$ be a strictly positive trace class operator on $\mathscr{H} \cdot \mathscr{D}\left(A^{-1 / 2}\right)$, the domain of $A^{-1 / 2}$, is dense in $\mathscr{H}$ and is a Hilbert space, denoted $\mathscr{H}(A)$, when equipped with the inner product

$$
\left\langle Y_{1}, Y_{2}\right\rangle_{A} \doteq\left\langle A^{-1 / 2} Y_{1}, A^{-1 / 2} Y_{2}\right\rangle
$$

Let us define

$$
I(Y) \doteq\left\{\begin{array}{cll}
(1 / 2)\langle Y, Y\rangle_{A}=(1 / 2)\left\|A^{-1 / 2} Y\right\|^{2} & \text { if } & Y \in \mathscr{D}\left(A^{-1 / 2}\right), \\
+\infty & \text { if } & Y \notin \mathscr{D}\left(A^{-1 / 2}\right),
\end{array}\right.
$$

and set

$$
G(Y) \doteq I(Y)+F(Y) .
$$

Thus on $\mathscr{H}(A), G(Y)=(1 / 2)\langle Y, Y\rangle_{A}+F(Y)$ and is therefore a $C^{\infty}$ Frechet differentiable functional on $\mathscr{H}(A)$. We have on $\mathscr{H}(A)$

$$
\begin{aligned}
D G(\bar{Y}) Y_{1} & =\left\langle\bar{Y}, Y_{1}\right\rangle_{A}+\left\langle F^{\prime}(\bar{Y}), Y_{1}\right\rangle=\left\langle\bar{Y}+A F^{\prime}(\bar{Y}), Y_{1}\right\rangle_{A}, \\
D^{2} G(\bar{Y})\left(Y_{1}, Y_{2}\right) & =\left\langle Y_{1}, Y_{2}\right\rangle_{A}+\left\langle F^{\prime \prime}(\bar{Y}) Y_{1}, Y_{2}\right\rangle=\left\langle Y_{1}+A F^{\prime \prime}(\bar{Y}) Y_{1}, Y_{2}\right\rangle_{A} \\
D^{j} G(\bar{Y})\left(Y_{1}, \ldots, Y_{j}\right) & \left.=D^{j} F(\bar{Y})\left(Y_{1}, \ldots, Y_{j}\right), j\right\rangle 2 .
\end{aligned}
$$

Although $G$ is not Frechet differentiable on $\mathscr{H}$, if $\bar{Y} \in \mathscr{D}\left(A^{-1}\right)$ we will use the notation

$$
\begin{aligned}
& G^{\prime}(\bar{Y}) \doteq A^{-1} \bar{Y}+F^{\prime}(\bar{Y}), \\
& G^{\prime \prime}(\bar{Y}) \doteq A^{-1}+F^{\prime \prime}(\bar{Y}) .
\end{aligned}
$$

Thus, the domain of $G^{\prime \prime}(Y)$ is $\mathscr{D}\left(A^{-1}\right)$.

A point $x \in \mathscr{H}$ is called a minimum point of $G$ if $G(x)=\inf \{G(Y) \mid Y \in \mathscr{H}\}$. Throughout this paper, if $K$ is a submanifold of $\mathscr{H}$, we will use the natural identification of $K_{x}$ with a subspace of $\mathscr{H}$ and set $N_{x} \doteq K_{x}^{\perp}$ in $\mathscr{H}$.

Lemma 1. Let $F$ satisfy the bound (1.2). Then we have the following facts. 
(a) The minimum points of G form a non-empty compact set.

(b) If $x$ is a minimum point of $G$, then $x \in \mathscr{D}\left(A^{-1}\right)$,

$$
G^{\prime}(x) \doteq A^{-1} x+F^{\prime}(x)=0,
$$

and

$$
G^{\prime \prime}(x) \geqq 0 .
$$

(c) If the set of minimum points of $G$ forms a smooth submanifold $M$ of $\mathscr{H}$, then $M_{x} \subset \mathscr{D}\left(A^{-1}\right)$,

$$
G^{\prime \prime}(x) v=0, \text { all } x \in M, \quad v \in M_{x},
$$

and $M$ is finite dimensional.

Proof. a) This follows from the facts that $G$ is lower semicontinuous on $\mathscr{H}(A)$ and that as $\|Y\| \rightarrow \infty, Y \in \mathscr{H}(A), G(Y) \rightarrow \infty$ like $\left\|A^{-1 / 2} Y\right\|^{2}$. For details, see [Ellis-Rosen (1); Lemma 3.1].

b) The bound (1.2) implies $x \in \mathscr{D}\left(A^{-1 / 2}\right)=\mathscr{H}(A)$.

Thus $x$ is also a minimum point of the smooth functional $G$ on $\mathscr{H}(A)$, so $D G(x) Y=0$ for all $Y \in \mathscr{H}(A)$. By $(2.3)$

$$
x+A F^{\prime}(x)=0 .
$$

This shows $x \in \mathscr{D}\left(A^{-1}\right)$ and proves (2.5). Similarly $D^{2} G(x) Y^{2} \geqq 0$ for all $Y \in \mathscr{H}(A)$, so by (2.3)

$$
\left\langle A^{-1 / 2} Y, A^{-1 / 2} Y\right\rangle+\left\langle F^{\prime \prime}(x) Y, Y\right\rangle=\langle Y, Y\rangle_{A}+\left\langle F^{\prime \prime}(x) Y, Y\right\rangle \geqq 0,
$$

and in particular for $Y \in \mathscr{D}\left(A^{-1}\right)$

$$
\left\langle\left(A^{-1}+F^{\prime \prime}(x)\right) Y, Y\right\rangle \geqq 0 .
$$

This is (2.6).

c) If $v \in M_{x}$, let $x_{t}$ be a smooth curve on $M$ with $x_{0}=x, x_{0}^{\prime}=v$. By (2.8)

$$
x_{t}+A F^{\prime}\left(x_{t}\right)=0 \text {. }
$$

Differentiating and then setting $t=0$ gives

$$
v+A F^{\prime \prime}(x) v=0 .
$$

Thus $v \in \mathscr{D}\left(A^{-1}\right)$ and $G^{\prime \prime}(x) v=A^{-1} v+F^{\prime \prime}(x) v=0$, proving (2.7).

We now prove that $M$ is finite dimensional. Since $A$ is trace class, (2.9) shows that each $x$ in $M$ the unit ball in $M_{x}$ is compact; hence $M_{x}$ is finite dimensional. Thus $\operatorname{dim}(M)<\infty$.

Lemma 2. Let $A$ be a strictly positive trace class operator on $\mathscr{H}$ and $V \subseteq \mathscr{D}\left(A^{-1}\right)$ a finite dimensional subspace. Then

$$
A^{V^{\perp}} \doteq\left(\pi_{V_{\perp}} A^{-1} \pi_{V_{\perp}}\right)^{-1}
$$

defines a strictly positive trace class operator on $V^{\perp}\left(A^{V^{\perp}}=0\right.$ on $\left.V\right)$. Therefore $A^{V^{\perp}}$ is the covariance operator of a mean zero Gaussian measure $P_{\left(A^{V^{\perp}}\right)}$.

Proof. In terms of the decomposition $\mathscr{H}=V \oplus V^{\perp}$, we can write the positive 
operators $A^{-1}, A$ as

$$
A^{-1}=\left(\begin{array}{c|c}
\alpha & \beta^{*} \\
\hline \beta & \gamma
\end{array}\right) \text { on } \mathscr{D}\left(A^{-1}\right), \quad A=\left(\begin{array}{c|c}
R & S^{*} \\
\hline S & T
\end{array}\right),
$$

so that, e.g., $\gamma=\pi_{V^{\perp}} A^{-1} \pi_{V^{\perp}}, R=\pi_{V} A \pi_{V}$. The identity $A^{-1} A=I$ yields

$$
\begin{aligned}
\alpha R+\beta^{*} S & =I_{V}, \\
\beta R+\gamma S & =0_{V}, \\
\beta S^{*}+\gamma T & =I_{V^{\perp}},
\end{aligned}
$$

where $I_{V}, 0_{V}$ denote the identity and zero operators on $V$ and $I_{V^{\perp}}$ denotes the identity operator on $V^{\perp}$.

$R=\pi_{V} A \pi_{V}$ is a bounded linear operator on the finite dimensional subspace $V$, and the strict positivity of $A$ implies $\operatorname{ker}(R)=\{0\}$. Thus $R$ has a bounded inverse and from $(2.12 \mathrm{~b})$ we have $\beta=-\gamma S R^{-1}$. Substituting this into (2.12c) shows

$$
\gamma\left(T-S R^{-1} S^{*}\right)=I_{V^{\perp}}
$$

Working similarly with the identity $A A^{-1}=I$ on $\mathscr{D}\left(A^{-1}\right)$, we find

$$
\left(T-S R^{-1} S^{*}\right) \gamma=I_{V^{\perp}} \text { on } \mathscr{D}\left(A^{-1}\right) \cap V^{\perp} \text {. }
$$

The last two equations, (2.13) and (2.14), show that $\gamma \doteq \pi_{V^{\perp}} A^{-1} \pi_{V^{\perp}}$, with domain $\mathscr{D}\left(A^{-1}\right) \cap V^{\perp}$, is invertible with inverse $T-S R^{-1} S^{*}$. Thus

$$
A^{V^{\perp}} \doteq\left(\pi_{V^{\perp}} A^{-1} \pi_{V^{\perp}}\right)^{-1}=\gamma^{-1}
$$

is well defined and we have

$$
A^{V^{\perp}}=T-S R^{-1} S^{*}
$$

This shows that $A^{V^{\perp}}$ is a bounded self-adjoint operator. $A^{V^{\perp}}$ is positive, since it is the inverse of a positive operator, and strictly positive by (2.13). $A^{V^{\perp}}$ is trace class since $T$ and $S$ are both trace class and $R^{-1}, S$ are both bounded [Simon (2); Chap. 2]. The last assertion in the theorem follows from [Gihman-Skorohod; Theorem V.6.1].

Let us record one additional fact. In case $\mathscr{H}$ itself is finite dimensional, the identity

$$
\left(\begin{array}{c|c}
I & 0 \\
\hline-S R^{-1} & I
\end{array}\right)\left(\begin{array}{c|c}
R & S^{*} \\
\hline S & T
\end{array}\right)=\left(\begin{array}{c|c}
R & S^{*} \\
\hline 0 & T-S R^{-1} S^{*}
\end{array}\right)
$$

shows that, by (2.11) and (2.15),

$$
\begin{aligned}
\operatorname{det}(A) & =\operatorname{det}(R) \operatorname{det}\left(T-S R^{-1} S^{*}\right) \\
& =\operatorname{det}\left(A_{V}\right) \operatorname{det}\left(A^{V^{\perp}}\right) .
\end{aligned}
$$

\section{Proof of Theorem 1.}

We first explain the main idea behind Theorem 1 . When $M$ is a point, Theorem 1 is proven in [Ellis-Rosen (1)]. In the general case, where $M$ is a smooth non- 
degenerate $m$-dimensional manifold, we use normal coordinates for $Y / \sqrt{n}$ in a neighborhood of $M$ :

$$
Y / \sqrt{n}=x+z / \sqrt{n}, \quad x \in M, \quad z \in N_{x} .
$$

We will see that the main contribution to (1.1) comes from such a neighborhood. The change of variables (3.1) in (1.3) gives formally

$$
\begin{aligned}
J_{n} & =\int \psi(Y / \sqrt{n}) \exp (-G(Y / \sqrt{n})) d Y \\
& =n^{m / 2} \int_{M}\left\{\int_{N_{x}} \psi(x+z / \sqrt{n}) I_{M}(x, z / \sqrt{n}) \exp (-n G(x+z / \sqrt{n})) d z\right\} d V_{M}(x),
\end{aligned}
$$

where $m \doteq \operatorname{dim}(M)$ and $I_{M}$, defined in (1.10), is the factor arising from our change of variables.

For each fixed $x \in M$, the inner integral

$$
J_{n}(x) \doteq \int_{N_{x}} \psi(x+z / \sqrt{n}) I_{M}(x, z / \sqrt{n}) \exp (-n G(x+z / \sqrt{n})) d z
$$

is itself precisely of the form (1.3), with $G(Y / \sqrt{n})$ replaced by $\overline{\mathscr{G}}(z / \sqrt{n})=$ $G(x+z / \sqrt{n})$ and the Hilbert space $\mathscr{H}$ replaced by $N_{x}$. Our assumption (1.6) that $M$ be nondegenerate means that $\overline{\mathscr{G}}(z)$ has a unique and nondegenerate minimum point, the vector 0 , in $N_{x}$. We are thus reduced to the case where $M$ is a point. This is the main idea behind our proof. Our greatest difficulty in proving Theorem 1 will be in justifying the change of variables (3.1) in our integral.

Proof of Theorem 1. To help keep the proof clear, we consign certain parts to a series of lemmas, which are proved in Sect. 4.

Let $\left\{e_{1}, e_{2}, \ldots\right\}$ be a basis of $\mathscr{H}$ formed by eigenvectors of $A$, and set $\mathscr{H}_{j} \doteq$ $\operatorname{span}\left\{e_{1}, \ldots, e_{j}\right\} . \mathscr{H}_{j}$ is an $A$-invariant subspace of $\mathscr{H}$ with $\mathscr{H}_{j} \subset \mathscr{H}_{j+1}$, and $\mathscr{H}=\bar{\cup} \mathscr{H}_{j}$. We define $\pi_{j} \doteq \pi_{\mathscr{H}_{j}}$ and $S_{j} \doteq \pi_{j} S$ for any subset $S \subseteq \mathscr{H}$.

For any subset $S \subseteq \mathscr{H}$, let $S^{\varepsilon} \doteq\left\{x \mid \inf _{Y \in S}\|Y-x\|<\varepsilon\right\}$, i.e., the vectors within $\varepsilon$ of $S$. Let $K$ be a finite dimensional submanifold of $\mathscr{H}$. We say that $K^{\delta}$ is a tubular neighborhood of $K$ if every $Y \in K^{\delta}$ can be written $Y=x+z$ for unique $x \in K$, $z \in N_{x}(K),\|z\|<\delta$. Here $N_{x}(K)$ denotes the space of normal vectors to $K$ at $x$. It is known that for compact $K$ we can find $\delta>0$ sufficiently small such that $K^{\delta}$ is a tubular neighborhood of $K$ [Guillemin-Pollack, p. 69-76]. It is easy to verify that for $j$ sufficiently large, $K_{j}$ is also a submanifold of $\mathscr{H}$. We say that $K^{\delta}$ is a uniformly tubular neighborhood of $K$ if $K^{\delta}$ is a tubular neighborhood of $K$ and $\left(K_{j}\right)^{\delta}$ is a tubular neighborhood of $K_{j}$ for all $j$ sufficiently large. It is not hard to modify the proof of the existence of tubular neighborhoods to show that if $K$ is compact, then we can find $\delta>0$ sufficiently small such that $K^{\delta}$ is a uniformly tubular neighborhood of $K$. Note, then, that the same is true for $K^{\delta^{\prime}}$ for any $\delta^{\prime}<\delta$.

As mentioned, the heart of our proof is the following lemma, concerning a nonlinear change of variables in a functional integral.

Warning on notation. In Lemma 3 and for the rest of the paper, if $V$ is a subspace of $\mathscr{H}$, then we write $P_{V}$ for the Gaussian measure $P_{\left(A^{V}\right)}$. Whenever the variable 
of integration is omitted in an integral with respect to $P_{V}$, that variable is understood to be $z$. In such an integral, the region of integration is understood to be $V$.

Lemma 3. Let $K$ be a compact, finite dimensional, submanifold of $\mathscr{H}$, with $K^{\delta}$ a uniformly tubular neighborhood of $K$. If $A^{-1} x$ is a smooth $\mathscr{H}$-valued function of $x$ on $K$, then $A^{N_{x}(K)}$ is a strictly positive trace class operator on $N_{x}(K)$ and for any bounded, uniformly continuous functional $\phi$ on $\mathscr{H}$ with supp $\phi \subseteq K^{\delta / 2}$,

$$
\begin{aligned}
\int \phi(Y) d P_{A}(Y)= & \int_{K}\left\{\int_{\|z\|<\delta} \phi(x+z) I_{K}(x, z) \exp \left(-\left\langle A^{-1} x, x\right\rangle / 2-\left\langle A^{-1} x, z\right\rangle\right) .\right. \\
& \left.\cdot d P_{N_{x}(K)}\right\}\left(\operatorname{det}\left(2 \pi A_{K_{x}}\right)\right)^{-1 / 2} d V_{K}(x)
\end{aligned}
$$

where $I_{K}$ and $d V_{K}$ are defined as in the statement of Theorem 1.

Let us now turn to $M$, the set of minimum points of $G$. By Lemma $1, M$ is a compact finite dimensional submanifold of $\mathscr{H}$. We can therefore find $\delta>0$ sufficiently small such that $M^{\delta}$ is a uniformly tubular neighborhood of $M$.

Let $f(Y)$ be a bounded, uniformly continuous functional, with $f=1$ on $M^{\delta / 4}$ and supp $f \subseteq M^{\delta / 2}$. The main contributions to (1.1) come from $Y / \sqrt{n} \in M^{\delta / 4}$. More precisely, by Lemma 4.1 of [Ellis-Rosen (1)] (which is based on [Varadhan; Sect. 3]) we can find a $\delta^{\prime}>0$ such that for any $\delta \leqq \delta^{\prime}$ and $f$ as above

$$
e^{n G^{*}} J_{n}=e^{n G^{*}} \int f(Y / \sqrt{n}) \psi(Y / \sqrt{n}) \exp (-n F(Y / \sqrt{n})) d P_{A}(Y)+O\left(e^{-n c}\right),
$$

for some $c=c(\delta)>0$. We therefore need only study the asymptotic expansion of the integral with the cutoff function $f$. At the end of our proof we will see how to remove this cutoff.

If $S \subseteq \mathscr{H}$ is a subset, we use the notation $\sqrt{n} S \doteq\{Y \mid Y=\sqrt{n} x$, for some $x \in S\}$. We will apply Lemma 3 to the functional $\phi(Y) \doteq f(Y / \sqrt{n}) \psi(Y / \sqrt{n})$. $\exp (-n F(Y / \sqrt{n}))$ and the compact submanifold $K \doteq \sqrt{n} M$. Note that because of the cutoff function $f(Y / \sqrt{n})$, supp $\phi \subseteq \sqrt{n}\left(M^{\delta / 2}\right)=(\sqrt{n} M)^{\sqrt{ } n \delta / 2}$. Since $M^{\delta}$ is a uniformly tubular neighborhood of $M$, it is easy to see that $(\sqrt{n} M)^{\sqrt{ } n \delta}$ is a uniformly tubular neighborhood of $\sqrt{n} M$. Also, since by (2.5)

$$
A^{-1} x=-F^{\prime}(x), \text { for all } x \in M,
$$

$A^{-1} x$ is smooth on $\sqrt{n} M$. Finally the condition that $\phi$ be bounded and uniformly continuous follows from the smoothness of $\psi$ and $F$ and the compactness of $M$. Since this compactness argument will be used repeatedly, we state it as a lemma, whose simple proof we omit.

Lemma 4. Let $K$ be a compact subset of $\mathscr{H}$ and $\phi$ a continuous functional on $\mathscr{H}$. Then for some $\delta>0, \phi$ is bounded on $K^{\delta}$.

We choose $\delta$ as small as required by Lemma 4. Then (3.5) and Lemma 3 yield

$$
e^{n G^{*}} J_{n}=e^{n G^{*}} \int_{\sqrt{ } n M}\left\{\int_{\|z / \sqrt{ } n\|<\delta}\left(\operatorname{det}\left(2 \pi A_{(\sqrt{ } n M)_{x}}\right)\right)^{-1 / 2} f\left(\frac{x+z}{\sqrt{n}}\right) \psi\left(\frac{x+z}{\sqrt{n}}\right) .\right.
$$




$$
\begin{aligned}
& \cdot \exp \left(-n F\left(\frac{x+z}{\sqrt{n}}\right)\right) I_{\sqrt{ } n M}(x, z) . \\
& \left.\cdot \exp \left(-\left\langle A^{-1} x, x\right\rangle / 2-\left\langle A^{-1} x, z\right\rangle\right) d P_{N_{x}(\sqrt{ } n M)}\right\} d V_{(\sqrt{ } n M)}(x) \\
& +O\left(e^{-n c}\right) .
\end{aligned}
$$

We intend to simplify the integral in (3.6) and obtain

$$
\begin{aligned}
e^{n G^{*}} J_{n}= & n^{m / 2} \int_{M}\left\{\int_{\|z / \sqrt{ } n\|<\delta} f(x+z / \sqrt{n}) \psi(x+z / \sqrt{n}) I_{M}(x, z / \sqrt{n})\right. \\
& \left.\cdot \exp \left(-n F_{3}(x, z / \sqrt{n})\right) d P_{B(x)}(z)\right\} c(x) d V_{M}(x)+O\left(e^{-n c}\right),
\end{aligned}
$$

where $F_{3}, c(x), B(x)$ are defined in Theorem 1 and $m \doteq \operatorname{dim}(M)$. (3.7) should be compared to (1.9).

To prove (3.7), we first note the following.

Lemma 5. For any integrable functional $h$ on $\sqrt{n} M$,

$$
\int_{\sqrt{ } n M} h(x) d V_{\sqrt{ } n M}(x)=n^{m / 2} \int_{M} h(\sqrt{n} x) d V_{M}(x) .
$$

Lemma 5 applied to (3.6) yields

$$
\begin{aligned}
e^{n G^{*}} J_{n}= & n^{m / 2} e^{n G^{*}} \int_{M}\left\{\int_{\|z / \sqrt{ } n\|<\delta} f(x+z / \sqrt{n}) \psi(x+z / \sqrt{n}) .\right. \\
& \cdot \exp (-n F(x+z / \sqrt{n})) I_{\sqrt{ } n M}(\sqrt{n} x, z)\left(\operatorname{det}\left(2 \pi A_{(\sqrt{n} M) \sqrt{n} x}\right)\right)^{-1 / 2} . \\
& \left.\cdot \exp \left(-n\left\langle A^{-1} x, x\right\rangle / 2-n\left\langle A^{-1} x, z / \sqrt{n}\right\rangle\right) d P_{N_{\sqrt{n} x}(\sqrt{n} M)}\right\} d V_{M}(x) \\
& +O\left(e^{-n c}\right) .
\end{aligned}
$$

Now, we note that $(\sqrt{n} M)_{\sqrt{n} x}=\sqrt{n}\left(M_{x}\right)=M_{x}$ since $M_{x}$ is a subspace, and $N_{\sqrt{ } n x}(\sqrt{n} M)=N_{x}(M)$. We also have the following fact.

Lemma 6. $I_{\sqrt{n} M}(\sqrt{n} x, z)=I_{M}(x, z / \sqrt{n})$.

We apply to (3.8) these facts, together with (see (2.5))

$$
\begin{aligned}
& G^{*}=G(x)=\left\langle A^{-1} x, x\right\rangle / 2+F(x), \\
& A^{-1} x+F^{\prime}(x)=G^{\prime}(x)=0 .
\end{aligned}
$$

We find

$$
\begin{aligned}
e^{n G^{*}} J_{n}= & n^{m / 2} \int_{M}\left\{\int_{\|z / \sqrt{ } n\|<\delta} f(x+z / \sqrt{n})\left(\operatorname{det}\left(2 \pi A_{M_{x}}\right)\right)^{-1 / 2} \psi(x+z / \sqrt{n}) I_{M}(x, z / \sqrt{n}) .\right. \\
& \left.\cdot \exp \left(-n F_{3}(x, z / \sqrt{n})\right) \exp \left(-\left\langle F^{\prime \prime}(x) z, z\right\rangle / 2\right) d P_{N_{x}(M)}\right\} d V_{M}(x)+O\left(e^{-n c}\right) .
\end{aligned}
$$

We now apply Lemma 4.4 of [Ellis-Rosen] with

$$
\mathscr{H}_{1}=N_{x x}(M), A_{1}=A^{N_{x}(M)} \text { and } \Lambda=\left(F^{\prime \prime}(x)\right)_{N_{x}(M)} .
$$


The condition of that lemma, that $A_{1}^{-1}+\Lambda=\left(G^{\prime \prime}(x)\right)_{N_{x}(M)}>0$, is precisely the nondegeneracy condition (1.6). The lemma tells us that $\operatorname{det}\left(I+A^{N_{x}(M)}\left(F^{\prime \prime}(x)\right)_{N_{x}(M)}\right)$ is well defined and positive, that $B(x) \doteq\left(G^{\prime \prime}(x)\right)_{N_{x}(M)}$ is the covariance operator of a mean zero Gaussian measure $P_{B(x)}$ on $N_{x}(M)$, and that

$$
d P_{B(x)}(z)=\left(\operatorname{det}\left(I+A^{N_{x}(M)}\left(F^{\prime \prime}(x)\right)_{N_{x}(M)}\right)\right)^{1 / 2} \exp \left(-\left\langle F^{\prime \prime}(x) z, z\right\rangle / 2\right) d P_{N_{x}(M)} .
$$

Applying this to (3.9) and recalling the definition of $c(x)$ in (1.13), we see that $c(x)$ is well defined and (3.7) holds.

We will complete the proof of Theorem 1 by expanding the terms $\psi, I_{M}$, and $e^{-n F_{3}}$ in (3.7) in powers of $n^{-1 / 2}$ up to a given order, bounding the contributions of the remainder, and then showing how to remove the cutoff function $f$. Our analysis will resemble that of the proof of Theorem 2.1 of [Ellis-Rosen (1)]. For each given order, we may need to choose a different $\delta$. We then choose $f$ in accordance with this $\delta$. Each time it appears, the letter $c$ will denote a (possibly different) constant independent of $n$.

Let $j$ be a fixed integer. For $\delta$ sufficiently small, we have in (3.7)

$$
\begin{aligned}
\psi(x+z / \sqrt{n}) & =\sum_{i=0}^{j} \frac{D^{i} \psi(x)}{i !}(z / \sqrt{n})^{i}+R_{1}(x, z / \sqrt{n}), \\
-n F_{3}(x, z / \sqrt{n}) & =-\sum_{i=3}^{j+2} n \frac{D^{i} F(x)}{i !}(z / \sqrt{n})^{i}+R_{2}(x, z / \sqrt{n}),
\end{aligned}
$$

with

$$
\begin{aligned}
& \left|R_{1}(x, z / \sqrt{n})\right| \leqq c(\|z\| / \sqrt{n})^{j+1}, \\
& \left|R_{2}(x, z / \sqrt{n})\right| \leqq c n(\|z\| / \sqrt{n})^{j+3},
\end{aligned}
$$

uniformly in $x \in M$. This follows from Lemma 4 applied to the error term in Taylor's theorem.

For any real number $\mu$ we have

$$
e^{\mu}=\sum_{i=0}^{j} \mu^{i} / i !+S_{j}(\mu), \quad \text { with }\left|S_{j}(\mu)\right| \leqq e^{\mu}|\mu|^{j+1},
$$

so

$$
\exp \left(-n F_{3}(x, z / \sqrt{n})\right)=\sum_{i=0}^{j}\left(-n F_{3}(x, z / \sqrt{n})^{i} / i !+S_{j}\left(-n F_{3}(x, z / \sqrt{n})\right),\right.
$$

with

$$
\begin{aligned}
S_{j}\left(-n F_{3}(x, z / \sqrt{n})\right) & \leqq \exp \left(\left|n F_{3}(x, z / \sqrt{n})\right|\right)\left|n F_{3}(x, z / \sqrt{n})\right|^{j+1} \\
& \leqq c\left(\|z\|^{3} / \sqrt{n}\right)^{j+1} \exp \left(\left|n F_{3}(x, z / \sqrt{n})\right|\right)
\end{aligned}
$$

uniformly in $x \in M$, by Lemma 4 .

Similarly expanding $I_{M}(x, z / \sqrt{n})$, we can write 


$$
\psi(x+z / \sqrt{n}) I_{M}(x, z / \sqrt{n}) \exp \left(-n F_{3}(x, z / \sqrt{n})\right)=Q_{j}(x, z / \sqrt{n})+R_{j}(x, z / \sqrt{n}) .
$$

Here $Q_{j}$ is the sum of all terms containing factors of $n^{-1 / 2}$ up to order $n^{-j / 2}$ and

$$
\left|R_{j}(x, z / \sqrt{n})\right| \leqq c n^{-(j+1) / 2}\left(1+\|z\|^{N}\right) \exp \left(n\left|F_{3}(x, z / \sqrt{n})\right|\right)
$$

for some $N$, uniformly in $x \in M$, again by Lemma 4 .

We can choose $\delta$ so small that for given $b>0$

$$
\left|n F_{3}(x, z / \sqrt{n})\right| \leqq c n(\|z\| / \sqrt{n})^{3} \leqq \delta c\|z\|^{2} \leqq(b / 4)\|z\|^{2}
$$

uniformly in $x \in M$, by Lemma 4 . Taking $b$ to be the constant appearing in the next lemma, we see from (3.10) that the contribution of $R_{j}$ to the inner integral in (3.7) is $O\left(n^{-(j+1) / 2}\right)$, uniformly in $x \in M$.

Lemma 7. There exist $b>0, d<\infty$, independent of $x \in M$, such that

$$
\int e^{b\|z\|^{2} / 2} d P_{B(x)}(z) \leqq d,
$$

and for all $a>0$ and $x \in M$

$$
P_{B(x)}\{z \mid\|z\| \geqq a\} \leqq d e^{-b a^{2} / 2}
$$

We will see in Lemma 8 that $c(x)$ is continuous and therefore bounded on $M$. We have thus shown

$$
\begin{aligned}
e^{n G^{*}} J_{n}= & n^{m / 2} \int_{M}\left\{\int_{N_{x}(M) \cap\{z \mid\|z / \sqrt{n}\|<\delta\}} f(x+z / \sqrt{n}) Q_{j}(x, z / \sqrt{n}) d P_{B(x)}(z)\right\} c(x) d V_{M}(x) \\
& +O\left(n^{m / 2-(j+1) / 2}\right) .
\end{aligned}
$$

The proof of Theorem 1 will be complete once we have shown that (3.13) is still valid if we replace $f$ by 1 and drop the restriction to $\{z \mid\|z / \sqrt{n}\|<\delta\}$. However, again by Lemma 4

$$
\left|Q_{j}(x, z / \sqrt{n})\right| \leqq c\left(1+\|z\|^{\bar{N}}\right) \text { for some } \bar{N}
$$

uniformly in $x \in M$ and all $z$. Since $f$ is bounded and $f(x+z / \sqrt{n})=1$ if $\|z\| / \sqrt{n}<$ $\delta / 4$, it will suffice to show that for some $\bar{\delta}>0$

$$
\int_{\{z \mid\|z\| / \sqrt{ } n \geqq \delta / 4\}}\left|Q_{j}(x, z / \sqrt{n})\right| d P_{B(x)}(z) \leqq c e^{-n \bar{\delta}}
$$

independent of $x \in M$. In view of (3.14), this follows from Lemma 7.

\section{Proofs of Lemmas 3-8}

Proof of Lemma 3. We will see that for finite dimensional $\mathscr{H}$ our lemma is a straightforward change of variables. In the general case, we will approximate $\mathscr{H}$ by the finite dimensional $\left\{\mathscr{H}_{j}\right\}$ and then pass to the limit $j \rightarrow \infty$. 
Let $k \doteq \operatorname{dim}(K)$. Since $K$ is compact and $\pi_{j} \rightarrow I$ strongly, it is easily checked that $K_{j} \doteq \pi_{j} K$ is a $k$-dimensional submanifold and

$$
\left(K_{j}\right)^{\delta} \supseteq K^{\delta / 2} \supseteq \operatorname{supp} \phi,
$$

for $j$ sufficiently large. Therefore

$$
\int \phi(Y) d P_{A}(Y)=\int_{\left(K_{j}\right)^{\delta}} \phi(Y) d P_{A}(Y) .
$$

We write $K_{j, x}$ and $N_{j, x}$ to denote, respectively, the tangent space and the normal space to $K_{j}$ in $\mathscr{H}$ at $x$. As usual we identify these with subspaces of $\mathscr{H}$.

$K_{j}$ is also a submanifold of $\mathscr{H}_{j}$. We will use $\widetilde{K}_{j, x}$ and $\tilde{N}_{j, x}$ to denote, respectively, the tangent space and the normal space to $K_{j}$ in $\mathscr{H}_{j}$ at $x$. Identifying these as usual with subspaces of $\mathscr{H}_{j} \subseteq \mathscr{H}$, we see that

$$
\begin{aligned}
& K_{j, x}=\tilde{K}_{j, x}, \\
& N_{j, x}=\tilde{N}_{j, x} \oplus \mathscr{H}_{j}^{\perp} .
\end{aligned}
$$

We also define $\left(\tilde{K}_{j}\right)^{\delta} \doteq\left\{x \in \mathscr{H}_{j} \mid \inf _{Y \in K_{j}}\|x-Y\|<\delta\right\}$, so that $\left(\tilde{K}_{j}\right)^{\delta}=\left(K_{j}\right)^{\delta} \cap \mathscr{H}_{j}$.

In terms of the decomposition $\mathscr{H}=\mathscr{H}_{j} \oplus \mathscr{H}_{j}^{\perp}, A$ has the matrix

$$
A=\left(\begin{array}{cc}
A_{\mathscr{H}_{j}} & 0 \\
0 & A_{\mathscr{H}}^{\frac{1}{j}}
\end{array}\right) .
$$

Because we are dealing with Gaussian probability measures, for any integrable $h$ we have

$$
\int_{H} h(Y) d P_{A}(Y)=\int_{\mathscr{H}_{j}^{\frac{1}{j}}}\left\{\int_{\mathscr{H}_{j} .} h(u+v) d P_{A_{\mathscr{H}_{j}}}(u)\right\} d P_{A_{\mathscr{H}_{j}^{\prime}}}(v) .
$$

Since $K_{j} \subseteq \mathscr{H}_{j}$, for any $v \in \mathscr{H}_{j}^{\perp}$ with $\|v\|<\delta$ and $x \in K_{j}, x+v$ is in $\left(K_{j}\right)^{\delta}$, so that by (4.6)

$$
\int_{\left(K_{j}\right)^{\delta}} \phi(Y) d P_{A}(Y)=\int_{\substack{v \in \mathscr{H}_{j}^{\perp} \\\{\|v\|<\delta\}}}\left\{\int_{\left(\widetilde{K}_{j}\right)^{\delta(v)}} \phi(u+v) d P_{A \mathscr{H} \mathcal{K}_{j}^{\perp}}(u)\right\} d P_{A \mathscr{H}_{j}^{\perp}}(v),
$$

where $\delta(v) \doteq \sqrt{\delta^{2}-\|v\|^{2}}$.

We can assume $j>k$ is so large that $\left(K_{j}\right)^{\delta}$ is a tubular neighborhood of $K_{j}$, and therefore $\left(\tilde{K}_{j}\right)^{\delta} \doteq\left(K_{j}\right)^{\delta} \cap \mathscr{H}_{j}$ is a tubular neighborhood of $K_{j}$ in $\mathscr{H}_{j}$. We will rewrite the inner integral in (4.7) using the following straightforward change of variables formula (for a proof, see [Weyl]):

$$
\int_{\left(\tilde{K}_{j}\right)^{\delta}} h(u) d^{j} u=\int_{\boldsymbol{K}_{j}}\left\{\int_{\substack{w \in \tilde{N}_{j}, x \\\|w\|<\delta}} h(x+w) I_{K_{j}}(x, w) d w^{j-k}\right\} d V_{K_{j}}(x)
$$

for any bounded continuous functional $h(u)$ on $\mathscr{H}_{j}$. In this formula, $I_{K_{j}}$ and $d V_{\boldsymbol{K}_{j}}$ are defined as in the statement of Theorem 1 , and $\mathscr{H}_{j}, \tilde{N}_{j, x}$ are viewed as $j$ and 
$j-k$ dimensional Hilbert spaces, respectively, with $d^{j} u$ and $d^{j-k} w$ denoting the natural Lebesque measures on these spaces.

To apply (4.8) to the inner integral of (4.7), note that as measures on $\mathscr{H}_{j}$

$$
\begin{aligned}
d P_{A \mathscr{H}_{j}}(u) & =\exp \left(-\left\langle A_{\mathscr{H}_{j}}^{-1} u, u\right\rangle / 2\right) d^{j} u /\left(\operatorname{det}\left(2 \pi A_{\mathscr{H}_{j}}\right)\right)^{1 / 2} \\
& =e^{-\left\langle A^{-1} u, u\right\rangle / 2} d^{j} u /\left(\operatorname{det}\left(2 \pi A_{\mathscr{H}_{j}}\right)\right)^{1 / 2} .
\end{aligned}
$$

Therefore, with $h(u) \doteq \phi(u+v) \exp \left(-\left\langle A^{-1} u, u\right\rangle / 2\right) /\left(\operatorname{det}\left(2 \pi A_{\mathscr{H}_{j}}\right)\right)^{1 / 2}$, (4.8) shows

$$
\begin{aligned}
\int_{\left(\tilde{K}_{j}\right) \delta(v)} \phi(u+v) d P_{A_{\mathscr{H}_{j}}}(u)= & \int_{K_{j}}\left\{\int_{\substack{\left.w \in \tilde{N}_{j}, x \\
\|w\|<\delta(v)\right\}}} \phi(x+w+v)\left(\operatorname{det}\left(2 \pi A_{\mathscr{H}_{j}}\right)\right)^{-1 / 2 .}\right. \\
& \left.\cdot \exp \left(-\left\langle A^{-1}(x+w), x+w\right\rangle / 2\right) I_{K_{j}}(x, w) d^{j-k_{w}}\right\} d V_{K_{j}}(x)
\end{aligned}
$$

The definition of $A^{\tilde{N}_{j, x}}$ shows that for $w \in \tilde{N}_{j, x}$

$$
\begin{aligned}
\left\langle A^{-1}(x+w), x+w\right\rangle / 2 & =\left\langle A^{-1} x, x\right\rangle / 2+\left\langle A^{-1} x, w\right\rangle+\left\langle A^{-1} w, w\right\rangle / 2 \\
& =\left\langle A^{-1} x, x\right\rangle / 2+\left\langle A^{-1} x, w\right\rangle+\left\langle\left(A^{\tilde{N}_{J, x}}\right)^{-1} w, w\right\rangle / 2 .
\end{aligned}
$$

Since as measures on $\tilde{N}_{j, x}$

$$
d P_{\tilde{N}_{j, x}}(w)=\exp \left(-\left\langle\left(A^{\tilde{N}_{j, x}}\right)^{-1} w, w\right\rangle / 2\right) d^{j-k} w /\left(\operatorname{det}\left(2 \pi A^{\tilde{N}_{j, x}}\right)\right)^{1 / 2},
$$

(4.9), (4.10), (4.11), and (2.16) show that

$$
\begin{aligned}
\int_{\left(\tilde{K_{j}}\right)^{\delta(v)}} \phi(u+v) d P_{A_{\mathscr{H}_{j}}}(u)= & \int_{K_{j}}\left\{\int_{\substack{\left.w \in N_{j, x} \\
\|w\|<\delta(v)\right\}}} \phi(x+w+v)\left(\operatorname{det}\left(2 \pi A_{K_{j, x}}\right)\right)^{-1 / 2} .\right. \\
& \left.\cdot \exp \left(-\left\langle A^{-1} x, x\right\rangle / 2-\left\langle A^{-1} x, w\right\rangle\right) I_{K_{j}}(x, w) d P_{\tilde{N}_{j, x}}(w)\right\} . \\
& \cdot d V_{K_{j}}(x) .
\end{aligned}
$$

We now show that (4.2), (4.7) and (4.12) can be combined to yield

$$
\begin{aligned}
\int \phi(Y) d P_{A}(Y)= & \int_{K_{j}}\left\{\int_{\|z\|<\delta} \phi(x+z)\left(\operatorname{det}\left(2 \pi A_{K_{j, x}}\right)\right)^{-1 / 2}\right. \\
& \left.\cdot \exp \left(-\left\langle A^{-1} x, x\right\rangle / 2-\left\langle A^{-1} x, z\right\rangle\right) I_{K_{j}}(x, z) d P_{N_{j, x}}\right\} d V_{K_{j}}(x) .
\end{aligned}
$$

To prove (4.13), first note that in the decomposition $\mathscr{H}=\mathscr{H}_{j} \oplus \mathscr{H}_{j}^{\perp}$ the matrix of $\pi_{N_{j, x}}$ is by (4.4)

$$
\pi_{N_{j, x}}=\left(\begin{array}{c|c}
\pi_{\tilde{N}_{j, x}} & 0 \\
\hline 0 & \pi_{\mathscr{H}_{j}^{\perp}}
\end{array}\right) .
$$


Therefore, on $\mathscr{D}\left(A^{-1}\right) \cap N_{j, x}=\tilde{N}_{j, x} \oplus\left(\mathscr{D}\left(A^{-1}\right) \cap \mathscr{H}_{j}^{\perp}\right)$

$$
\begin{aligned}
& \pi_{N_{j, x}} A^{-1} \pi_{N_{j, x}}=\left(\begin{array}{c|c}
\pi_{\tilde{N}_{j, x}} & 0 \\
\hline 0 & \pi_{\mathscr{H}_{j}^{\frac{1}{j}}}
\end{array}\right)\left(\begin{array}{c|c}
A_{\mathscr{H}_{j}}^{-1} & 0 \\
\hline 0 & A_{\mathscr{H}_{j}^{\frac{1}{j}}}^{-1}
\end{array}\right)\left(\begin{array}{c|c}
\pi_{\tilde{N}_{j, x}} & 0 \\
\hline 0 & \pi_{\mathscr{H}_{j}^{\frac{1}{j}}}
\end{array}\right) \\
& =\left(\begin{array}{c|c}
\pi_{\tilde{N}_{j, x}} A_{\mathscr{H}_{j}}^{-1} \pi_{\tilde{N}_{j, x}} & 0 \\
\hline 0 & A_{\mathscr{H}_{j}^{1}}^{-1}
\end{array}\right)=\left(\begin{array}{c|c}
\pi_{\tilde{N}_{j, x}} A^{-1} \pi_{\tilde{N}_{j, x}} & 0 \\
\hline 0 & \frac{0}{A_{\mathscr{H}_{j}^{\prime}}^{-1}}
\end{array}\right) .
\end{aligned}
$$

By Lemma 2 these operators are invertible, and we have

$$
A^{N_{j, x}}=\left(\begin{array}{ll}
A^{\tilde{N}_{j, x}} & 0 \\
0 & A_{\mathscr{H}_{j}^{\perp}}
\end{array}\right) .
$$

The operators in (4.15) are positive and trace class. Once again, because we are dealing with Gaussian probability measures, (4.15) shows that for any integrable $h$

$$
\int h(z) d P_{N_{j, x}}(z)=\int_{\mathscr{H}_{j}^{\perp}}\left\{\int_{\tilde{N}_{j, x}} h(w+v) d P_{\tilde{N}_{j, x}}(w)\right\} d P_{A_{\mathscr{H}_{j}^{\perp}}}(v) .
$$

Secondly, note that for $x \in \mathscr{H}_{j}, v \in \mathscr{H}_{j}^{\perp},\left\langle A^{-1} x, w\right\rangle=\left\langle A^{-1} x, w+v\right\rangle$, and since $K_{j} \subseteq \mathscr{H}_{j}$, the definition (1.10) of $I_{K_{j}}$ shows that $I_{K_{j}}(x, w)=I_{K_{j}}(x, w+v)$. Together with (4.16), (4.2), (4.7) and (4.12) now prove (4.13).

We wish to pass to the limit $j \rightarrow \infty$ in (4.13). We will do this in two steps. We write $N_{x}$ for $N_{x}(K)$ and $\chi_{\delta}(z)$ for the characteristic function of the set $\{z \mid\|z\|<\delta\}$. Assertion (a). For any $\varepsilon>0$, we can find a $J$ such that for all $j>J$ the right-hand side of (4.13) is within $\varepsilon$ of

$$
\begin{aligned}
& \int_{K}\left\{\int \phi(x+z) \chi_{\delta}(z)\left(\operatorname{det}\left(2 \pi A_{K_{x}}\right)\right)^{-1 / 2}\right. \\
& \left.\quad \cdot \exp \left(-\left\langle A^{-1} x, x\right\rangle / 2-\left\langle A^{-1} x, z\right\rangle\right) I_{K}\left(x, \pi_{N_{x}} z\right) d P_{N_{j, \pi_{j}}}\right\} d V_{K}(x) .
\end{aligned}
$$

Assertion (b). For each $x \in K, P_{N_{j, \pi} x} \rightarrow P_{N_{x}}$ weakly as measures on $\mathscr{H}$.

Let us show how these two assertions complete the proof of our lemma. The support property of $\phi$ expressed in (4.1) shows that the inner integrand in (4.17) is for each fixed $x$ a bounded continuous function of $z$. By Assertion (b), the inner integral in (4.17) converges to

$$
\int \phi(x+z) \chi_{\delta}(z)\left(\operatorname{det}\left(2 \pi A_{K_{x}}\right)\right)^{-1 / 2} \exp \left(-\left\langle A^{-1} x, x\right\rangle / 2-\left\langle A^{-1} x, z\right\rangle\right) I_{K}(x, z) d P_{N_{x}}(z) .
$$

Furthermore, the assumption that $A^{-1} x$ is a smooth function of $x$ on the compact set $K$, together with (4.26) of the following lemma, show that the inner integral can be bounded by a constant, independent of $j$. By the dominated convergence theorem, (4.17) converges to the right-hand side of (3.4). The proof of Lemma 3 follows immediately.

The next lemma collects some facts needed in the proof of Assertions (a) and (b).

Lemma 8. Let $\mathscr{B}$ denote the space of bounded linear operators on $\mathscr{H}$ with the uniform operator norm, $\mathscr{I}$ the subset of $\mathscr{B}$ consisting of trace class operators with the trace norm, and $\mathscr{H} \mathscr{S}$ the subset of $\mathscr{B}$ consisting of Hilbert-Schmidt operators with Hilbert- 
Schmidt norm. Then under the conditions of Lemma 3,

$$
\left.\begin{array}{ll}
\pi_{K_{j, \pi_{j} x}} & \rightarrow \pi_{K_{x}} \\
\pi_{N_{j, \pi_{j} x}} & \rightarrow \pi_{N_{x}} \\
A^{-1} \pi_{K_{J, \pi_{j} x}} & \rightarrow A^{-1} \pi_{K_{x}}
\end{array}\right\} \quad \begin{aligned}
& \\
& A^{N_{j, \pi_{j} x}} \rightarrow A^{N_{x}} \\
& \left(A^{\left.N_{j, \pi_{j} x}\right)^{1 / 2}} \rightarrow\left(A^{N_{x}}\right)^{1 / 2}\right.
\end{aligned} \quad \begin{aligned}
& \text { in } \mathscr{B} \text {, uniformly in } \mathscr{I}, \\
& \text { in } \mathscr{B} \text { and } \mathscr{H} \mathscr{S},
\end{aligned}
$$

$\pi_{K_{x}}$ and $\pi_{N_{x}}$ are continuous $\mathscr{B}$-valued functions on $K$,

$A^{N_{x}}$ is a continuous $\mathscr{I}$-valued and $\mathscr{B}$-valued function on $K$,

$\left(A^{N_{x}}\right)^{1 / 2}$ is a continuous $\mathscr{H} \mathscr{S}$-valued and $\mathscr{B}$-valued function on $K$,

$$
\left.\begin{array}{l}
\operatorname{det}\left(A_{K_{j, \pi_{j} x}}\right) \rightarrow \operatorname{det}\left(A_{K_{x}}\right) \\
\operatorname{det}\left(A^{K_{j, \pi_{j} x}}\right) \rightarrow \operatorname{det}\left(A^{K_{x}}\right)
\end{array}\right\} \quad \text { uniformly in } x \in K,
$$

$\operatorname{det}\left(A_{K_{x}}\right)$ is continuous and nonzero on $K$,

$c(x)$, defined in (1.13), is continuous on $M$.

Proof of Assertion (a). We will work locally. $K$ can be covered by a finite number of coordinate neighborhoods. Let $\mathscr{V}$ be such a neighborhood, and let $x(s), s \in \mathscr{U} \subseteq$ $R^{k}$, be a parametrization of $\mathscr{V}$. Then, $\pi_{j} x(s)$ is a parametrization of $\pi_{j} \mathscr{V} \subseteq K_{j}$. Setting $x_{i}(s)=\frac{\partial x(s)}{\partial s_{i}}, 1 \leqq i \leqq k$, and recalling the local definition of $d V_{K_{j}}$ [Spivak, Vol. I, p. 423], we see that the integral in (4.13) over the region $\pi_{j} \mathscr{V}$ can be written as

$$
\begin{aligned}
& \int_{\pi_{j} \mathscr{V}}\left\{\int_{\|z\|<\delta} \phi(x+z)\left(\operatorname{det}\left(2 \pi A_{K_{j, x}}\right)\right)^{-1 / 2} .\right. \\
& \left.\quad \cdot \exp \left(-\left\langle A^{-1} x, x\right\rangle / 2-\left\langle A^{-1} x, z\right\rangle\right) I_{K_{j}}(x, z) d P_{N_{j, x}}\right\} d V_{K_{j}}(x) \\
& =\int_{\mathscr{U}}\left\{\int_{\|z\|<\delta} \phi\left(\pi_{j} x(s)+z\right)\left(\operatorname{det}\left(2 \pi A_{K_{j, \pi_{j} x(s)}}\right)\right)^{-1 / 2} .\right. \\
& \left.\quad \cdot \exp \left[-\left\langle\pi_{j} A^{-1} x(s), x(s)\right\rangle / 2-\left\langle\pi_{j} A^{-1} x(s), z\right\rangle\right] I_{K_{j}}\left(\pi_{j} x(s), z\right) d P_{N_{j, \pi_{j} x(s)}}\right\} . \\
& \quad \cdot\left(\operatorname{det}\left(\left\langle\pi_{j} x_{i}(s), x_{\ell}(s)\right\rangle\right)\right)^{1 / 2} d^{k} \mathrm{~s} .
\end{aligned}
$$

Let us show that this is, for $j$ sufficiently large, within $\varepsilon$ of

$$
\begin{aligned}
& \int_{\mathscr{U}}\left\{\int_{\|z\|<\delta} \phi(x(s)+z)\left(\operatorname{det}\left(2 \pi A_{K_{x(s)}}\right)\right)^{-1 / 2} .\right. \\
& \left.\cdot \exp \left(-\left\langle A^{-1} x(s), x(s)\right\rangle / 2-\left\langle A^{-1} x(s), z\right\rangle\right) I_{K}\left(x(s), \pi_{N_{x(s)}} z\right) d P_{N_{j, \pi_{j} x(s)}}\right\} \cdot \\
& \cdot\left(\operatorname{det}\left(\left\langle x_{i}(s), x_{\ell}(s)\right\rangle\right)\right)^{1 / 2} d^{k} s=\int_{\mathscr{V}}\left\{\int_{\|z\|<\delta} \phi(x+z)\left(\operatorname{det}\left(2 \pi A_{K_{x}}\right)\right)^{-1 / 2} \cdot\right.
\end{aligned}
$$




$$
\left.\cdot \exp \left(-\left\langle A^{-1} x, x\right\rangle / 2-\left\langle A^{-1} x, z\right\rangle\right) I_{K}\left(x, \pi_{N_{x}} z\right) d P_{N_{j}, \pi_{j} x}\right\} d V_{K}(x)
$$

For this we will show that all terms in the integrands are uniformly bounded and corresponding terms are uniformly close. First note that $x(s) \in K$ and $\|z\|<\delta$. Since $\phi$ is bounded and uniformly continuous, $\phi\left(\pi_{j} x(s)+z\right)$ is bounded and close to $\phi(x(s)+z)$. Since $A^{-1} x$ is a smooth function of $x$ on $K,\left\langle\pi_{j} A^{-1} x(s), x(s)\right\rangle / 2+$ $\left\langle\pi_{j} A^{-1} x(s), z\right\rangle$ is bounded and close to $\left\langle A^{-1} x(s), x(s)\right\rangle / 2+\left\langle A^{-1} x(s), z\right\rangle$. Similarly $\operatorname{det}\left(\left\langle\pi_{j} x_{i}(s), x_{\ell}(s)\right\rangle\right)$ is bounded and close to $\operatorname{det}\left(\left\langle x_{i}(s), x_{\ell}(s)\right\rangle\right)$. The terms involving $\operatorname{det}\left(A_{K_{j, \pi} x(s)}\right)$ are handled by (4.24) and (4.26). Finally, by (4.19), $z=\pi_{N_{j}, \pi_{j} x(s)} z$ is close to $\pi_{N_{x(s)}} z$, and it is then easily seen from the definitions that $I_{K_{j}}\left(\pi_{j} x(s), z\right)$ is bounded and close to $I_{K}\left(x(s), \pi_{N_{x(s)}} z\right)$. Since, as mentioned, $K$ can be covered by a finite number of such $\mathscr{V}$, Assertion (a) is proven.

Proof of Assertion (b). By [Gihman-Skorohod, p. 379], this follows from (4.21b).

This completes the proof of Lemma 3.

Proof of Lemma 5. Let $\mathscr{V}$ be a coordinate neighborhood of $x$ in $M$, and let $x(s)$, $s \in \mathscr{U} \in R^{m}$, be a parametrization of $\mathscr{V}$. Set $x_{i}(s)=\frac{\partial x(s)}{\partial s_{i}}$, and $g_{i j}(s)=\left\langle x_{i}(s), x_{j}(s)\right\rangle$. In terms of this parametrization, the volume element of $M$ determined by $\mathscr{H}$ is

$$
\left(\operatorname{det}\left(g_{i j}(s)\right)\right)^{1 / 2} d s_{1} \ldots d s_{m} \text {. }
$$

We can use $\sqrt{n} x(s)$ as a parametrization of a neighborhood of $\sqrt{n} x$ in $\sqrt{n} M$. In terms of this parametrization the volume element of $\sqrt{n} M$ determined by $\mathscr{H}$ is

$$
n^{m / 2}\left(\operatorname{det} g_{i j}(s)\right)^{1 / 2} d s_{1} \ldots d s_{m},
$$

where $m \doteq \operatorname{dim}(M)$. This proves Lemma 5 .

Proof of Lemma 6. If $\tilde{v}(x)$ is the extension of $v$ used in the definition (1.8) of $W_{x, z}$ for $M$, we can take $\tilde{v}(x / \sqrt{n})$ as the extension to be used in the corresponding definition for $\sqrt{n} M$. Lemma 6 follows easily.

Proof of Lemma 7. By [Gihman-Skorohod; p. 351], we see that for each $x \in M$

$$
\int e^{b\|z\|^{2} / 2} d P_{B(x)}(z)=[\operatorname{det}(I-b B(x))]^{-1 / 2}>0
$$

for $b$ sufficiently small, and by [Ellis-Rosen (1); Lemma 4.4]

$$
B(x)=\left(A^{N_{x}}\right)^{1 / 2}\left[I+\left(A^{N_{x}}\right)^{1 / 2}\left(F^{\prime \prime}(x)_{N_{x}}\right)\left(A^{N_{x}}\right)^{1 / 2}\right]^{-1}\left(A^{N_{x}}\right)^{1 / 2} .
$$

(3.11) will follow from (4.29) once we show that $B(x)$ in (4.30) is a continuous $\mathscr{I}$ valued function on $M$ [Simon (2); p. 48]. Then (3.12) follows from (3.11) by Chebyshev's inequality.

Now by $(4.23 \mathrm{~b}),\left(A^{N_{x}}\right)^{1 / 2}$ is continuous in the Hilbert-Schmidt norm, so that it suffices to prove that the middle factor in (4.30) is continuous in the uniform operator norm [Simon (2); p. 31]. This follows from the smoothness of $F,(4.22)$, 
(4.23b), and the fact that inversion is a continuous map from $\mathscr{B}$ to $\mathscr{B}$ on the open set of invertible operators.

Proof of Lemma 8. We work locally. Let $\mathscr{V}$ be a coordinate neighborhood of $K$ and $x(s), s \in \mathscr{U} \in R^{k}$, a parametrization of $\mathscr{V}$. Then $\left\{\pi_{j} x(s), s \in \mathscr{U}\right\}$ is a parametrization $\pi_{j} \mathscr{V}$, a coordinate neighborhood of $K_{j} ;\left\{x_{i}(s) \doteq \frac{\partial x(s)}{\partial s_{i}}, i \leqq i \leqq k\right\}$ form a basis for $K_{x(s)} ;$ and for large $j,\left\{\pi_{j} x_{i}(s), 1 \leqq i \leqq k\right\}$ form a basis for $K_{j, \pi_{j} x(s)}$.

Define the $k \times k$ matrices, $g(s)$ and $g_{j}(s)$, by

$$
\begin{aligned}
(g(s))_{i \tau} & =\left\langle x_{i}(s), x_{\tau}(s)\right\rangle, \\
\left(g_{j}(s)\right)_{i \tau} & =\left\langle\pi_{j} x_{i}(s), x_{\tau}(s)\right\rangle .
\end{aligned}
$$

It is easily verified by checking on the above mentioned bases that

$$
\begin{aligned}
\pi_{K_{x(s)}} Y & =\sum_{i, \tau}\left(g^{-1}(s)\right)_{i \tau}\left\langle x_{\tau}(s), Y>x_{i}(s),\right. \\
\pi_{K_{j, \pi_{j} x(s)}} Y & =\sum_{i, \tau}\left(g_{j}^{-1}(s)\right)_{i \tau}\left\langle\pi_{j} x_{\tau}(s), Y>\pi_{j} x_{i}(s) .\right.
\end{aligned}
$$

These formulae prove (4.18) and also (4.19) and (4.22) since, e.g., $\pi_{N_{x}}=I-\pi_{M_{x}}$. They also show that

$$
\begin{gathered}
A^{-1} \pi_{K_{x(s)}} Y=\sum_{i, \tau}\left(g^{-1}(s)\right)_{i \tau}\left\langle x_{\tau}(s), Y\right\rangle A^{-1} x_{i}(s), \\
A^{-1} \pi_{K_{j, \pi_{j} x(s)}} Y=\sum_{i, \tau}\left(g_{j}^{-1}(s)\right)_{i \tau}\left\langle\pi_{j} x_{\tau}(s), Y\right\rangle \pi_{j} A^{-1} x_{i}(s) .
\end{gathered}
$$

Since by assumption $A^{-1} x$ is a smooth function of $x$ on $K$, these last formulae prove (4.20).

By (2.15) we have

$$
\begin{gathered}
A^{N_{x(s)}}=A_{N_{x(s)}}-\left(\pi_{N_{x(s)}} A \pi_{K_{x(s)}}\right)\left(A_{K_{x(s)}}\right)^{-1}\left(\pi_{N_{x(s)}} A \pi_{K_{x(s)}}\right)^{*} \\
A^{N_{j, \pi_{j} x(s)}}=A_{N_{j, \pi_{j} x(s)}}-\left(\pi_{N_{j, \pi_{j} \times(s)}} A \pi_{K_{j, \pi_{j} \times(s)}}\right)\left(A_{K_{j, \pi_{j} \times(s)}}\right)^{-1}\left(\pi_{N_{j, \pi_{j} \times(s)}} A \pi_{K_{j, \pi_{j} \times(s)}}\right)^{*} .
\end{gathered}
$$

Since inversion is a continuous map from $\mathscr{B}$ to $\mathscr{B}$ on the open set of invertible operators, (4.18), (4.19) and the fact that $A \in \mathscr{I}$, together with standard facts about $\mathscr{I}$ [Simon (2); Chap. 2], imply (4.21a). Similarly, using (4.22), we have (4.23a).

To prove the assertions about determinants, note that by (4.33)

$$
A_{K_{x(s)}} x_{\ell}(s)=\pi_{K_{x(s)}} A x_{\ell}(s)=\sum_{i, \tau}\left(g^{-1}(s)\right)_{i \tau}\left(x_{\tau}(s), A x_{\ell}(s)\right) x_{i}(s) .
$$

Thus, in the basis $\left\{x_{i}(s), 1 \leqq i \leqq k\right\}$

$$
\left(A_{K_{x(s)}}\right)_{\ell i}=\sum_{\tau}\left(g^{-1}(s)\right)_{i \tau}\left\langle x_{\tau}(s), A x_{\ell}(s)\right\rangle .
$$

Similarly in the basis $\pi_{j} x_{i}(s), 1 \leqq i \leqq k$, we find

$$
\left(A_{K_{j, \pi j} x(s)}\right)_{\ell i}=\sum_{\tau}\left(g_{j}^{-1}(s)\right)_{i \tau}\left\langle\pi_{j} x_{\tau}(s), A x_{\ell}(s)\right\rangle .
$$


This proves (4.24) and (4.26). The fact that $\operatorname{det}\left(A_{K_{x(s)}} \neq 0\right.$ follows from the strict positivity of $A$.

We similarly find formulas for the matrices of $\left(A^{K_{x(s)}}\right)^{-1}$ and $\left(A^{K_{j, \pi_{j} x(s)}}\right)^{-1}$. They look like (4.40) and (4.41) with $A$ replaced by $A^{-1}$. Since $A^{-1} x$ is a smooth function of $x$ on $K,(4.25)$ follows.

Facts (4.21b) and (4.23b) follow from (4.21a) and (4.23a), respectively [Simon (2); p. 42].

Finally, to prove the continuity of $c(x)$, we need only note (4.26), (4.23a), (4:22), and the smoothness of $F$ [Simon (2); p. 48]. (We have seen in our proof of (3.7) that for each $x$, the determinants appearing in the definition of $c(x)$ are nonzero.)

\section{Proofs of Theorems 2, 3, and 4}

Proof of Theorem 2. Using the notation of the proof of Theorem 1, by (3.7) we have

$$
\begin{aligned}
e^{n G^{*}} J_{n}= & n^{m / 2} \int_{M}\left\{\int_{N_{x}} f(x+z / \sqrt{n}) \psi(x+z / \sqrt{n}) I_{M}(x, z / \sqrt{n}) .\right. \\
& \left.\cdot \exp \left(-n F_{3}(x, z / \sqrt{n})\right) d P_{B(x)}(z)\right\} c(x) d V_{M}(x)+O\left(e^{-n c}\right) .
\end{aligned}
$$

Take any $x \in M$ and write it as $x=s x_{0}$ for some $s \in \mathscr{S}$. The inner integral is

$$
\begin{aligned}
& \int_{N_{s x_{0}}} f\left(s x_{0}+z / \sqrt{n}\right) \psi\left(s x_{0}+z / \sqrt{n}\right) I_{M}\left(s x_{0}, z / \sqrt{n}\right) \exp \left(-n F_{3}\left(s x_{0}, z / \sqrt{n}\right)\right) . \\
& \quad \cdot d P_{B\left(s x_{0}\right)}(z) .
\end{aligned}
$$

Let $\mathscr{H}_{1}$ and $\mathscr{H}_{2}$ be Hilbert spaces. In general, if $R$ is the covariance operator of a Gaussian probability measure $P_{R}$ on $\mathscr{H}_{1}$ and $U$ is a unitary operator from $\mathscr{H}_{1}$ to $\mathscr{H}_{2}$, we have for any integrable $h$ on $\mathscr{H}_{2}$

$$
\int_{\mathscr{H}_{1}} h(U Y) d P_{R}(Y)=\int_{\mathscr{H}_{2}} h(W) d P_{U R U^{-1}}(W) .
$$

In the present situation it is easily seen that $s$ is a unitary transformation from $N_{x_{0}}$ to $N_{s x_{0}}$, so that for any integrable $h$ on $N_{s x_{0}}$

$$
\int_{N_{x_{0}}} h(s z) d P_{B\left(x_{0}\right)}(z)=\int_{N_{s x_{0}}} h(z) d P_{s B\left(x_{0}\right) s^{-1}}(z) .
$$

However it is easily checked using (1.15) that

$$
s B\left(x_{0}\right) s^{-1}=B\left(s x_{0}\right) .
$$

Thus the integral (5.2) can be written as

$$
\begin{aligned}
& \int_{N_{x_{0}}} f\left(s x_{0}+s z / \sqrt{n}\right) \psi\left(s x_{0}+s z / \sqrt{n}\right) I_{M}\left(s x_{0}, s z / \sqrt{n}\right) . \\
& \cdot \exp \left(-n F_{3}\left(s x_{0}, s z / \sqrt{n}\right)\right) d P_{B\left(x_{0}\right)}(z) \\
= & \int_{N_{x_{0}}} f\left(s x_{0}+s z / \sqrt{n}\right) \psi\left(x_{0}+z / \sqrt{n}\right) I_{M}\left(x_{0}, z / \sqrt{n}\right) .
\end{aligned}
$$




$$
\cdot \exp \left(-n F_{3}\left(x_{0}, z / \sqrt{n}\right)\right) d P_{B\left(x_{0}\right)}(z)
$$

by the invariance of $J_{n}$ under $\mathscr{S},(1.15)$. Note that since $s$ is a unitary transformation of $N_{x_{0}}$ onto $N_{s x_{0}}, f\left(s x_{0}+s z / \sqrt{n}\right)$, as a function of $z \in N_{x_{0}}$, is equal to 1 for $\|z\| / \sqrt{n}<\delta / 4$ and zero for $\|z\| / \sqrt{n} \geqq \delta / 2$. The proof of Theorem 2 now follows as in the proof of Theorem 1.

Since $C^{\infty}(\mathscr{H})$ is dense in $C(\mathscr{H})$, it suffices to prove Theorem 3 and 4 for $\phi$ smooth.

Proof of Theorem 3. By (1.20) we have

$$
\begin{aligned}
& \int \phi(Y / n) d Q_{n}(Y) \\
& \quad=\int \phi(Y / \sqrt{n}) \exp (-n F(Y / \sqrt{n})) d P_{A}(Y) / \int \exp (-n F(Y / \sqrt{n})) d P_{A}(Y) .
\end{aligned}
$$

By Theorem 1,

$$
\begin{gathered}
e^{n G^{*}} \int \phi(Y / \sqrt{n}) \exp (-n F(Y / \sqrt{n})) d P_{A}(Y)=n^{m / 2} \int \phi(x) c(x) d V_{M}(x)+O\left(n^{m / 2-1}\right), \\
e^{n G^{*}} \int \exp (-n F(Y / \sqrt{n})) d P_{A}(Y)=n^{m / 2} \int c(x) d V_{M}(x)+O\left(n^{m / 2-1}\right),
\end{gathered}
$$

which combine to prove Theorem 3.

Proof of Theorem 4. By (1.20) we have

$$
\begin{aligned}
\int \phi\left(\frac{Y-n \pi(Y / n)}{\sqrt{n}}\right) d Q_{n}(Y)= & \int \phi(\sqrt{n}[Y / \sqrt{n}-\pi(Y / \sqrt{n})]) . \\
& \cdot \exp (-n F(Y / \sqrt{n})) d P_{A}(Y) / \int \exp (-n F(Y / \sqrt{n})) \cdot \\
& \cdot d P_{A}(Y) .
\end{aligned}
$$

By (3.7), in the notation of the proof of Theorem 1,

$$
\begin{aligned}
e^{n G^{*}} & \int \phi(\sqrt{n}[Y / \sqrt{n}-\pi(Y / \sqrt{n})]) \exp (-n F(Y / \sqrt{n})) d P_{A}(Y) \\
= & n^{m / 2} \int_{M}\left\{\int_{N_{x}} f(x+z / \sqrt{n}) \phi(z) I_{M}(x, z / \sqrt{n}) .\right. \\
& \left.\cdot \exp \left(-n F_{3}(x, z / \sqrt{n})\right) d P_{B(x)}(z)\right\} c(x) d V_{M}(x)+O\left(e^{-n c}\right),
\end{aligned}
$$

and the proof of Theorem 1 shows that this is equal to

$$
n^{m / 2} \int_{M}\left\{\int_{N_{x}} \phi(z) d P_{B(x)}(z)\right\} c(x) d V_{M}(x)+O\left(n^{m / 2-1}\right) .
$$

This, together with (5.9), proves Theorem 4.

\section{Extension to $\mathbf{C}[0,1]$}

In many applications, integrals of the form (1.1) arise where the functional $F$ is 
not defined on all of $L^{2}[0,1]$, but only on $C[0,1]$. In this section, we illustrate how our results can be extended to this setting by proving the $C[0,1]$-analogue of Theorem 1 . We denote $C[0,1]$ by $\mathscr{C}, L^{2}[0,1]$ by $\mathscr{H}$, and write the supremum norm of $\mathscr{C}$ as $\|-\|$; the norm of $\mathscr{H}$ will be written as $\|-\|_{2}$ and the inner product of $\mathscr{H}$ as $\langle-,-\rangle$. As above, "smooth" means $C^{\infty}$. Let $P_{A}$ be a mean zero Gaussian probability measure on $\mathscr{C}$ with covariance operator $A$ and covariance function $A(s, t)$. Let $\psi$ and $F$ be functionals on $\mathscr{C}$ and define the functional $G$ by (2.2). $G$ is finite on $\mathscr{D}\left(A^{-1 / 2}\right)$, which is a subset of $\mathscr{C}$ [Ellis-Rosen (2); Lemma 4.3(d)].

We next state the hypotheses under which the extension to $\mathscr{C}$ of Theorem 1 will be valid. The bound (6.2) below, which is weaker than (1.2), is condition $\left(d^{\prime}\right)$ in [Simon (2); p. 185]. This weaker bound is useful for applications.

Hypothesis 1 . $A$ is strictly positive on $\mathscr{H}$. Also,

$$
|A(s, t)| \leqq c<\infty,\left|A(s, t)-A\left(s^{\prime}, t\right)\right| \leqq \beta\left(\left|s-s^{\prime}\right|\right), \quad \text { all } 0 \leqq s, s^{\prime}, t \leqq 1,
$$

where $\beta$ is some function on $[0,1]$ which is nondecreasing, is continuous on $[0, \varepsilon]$, and satisfies $\beta(0)=0$ and $\int_{0}^{\varepsilon} \beta(u) u^{-1}[\log (1 / u)]^{-1 / 2} d u<\infty$ for some $\varepsilon \in(0,1 / e)$ (e.g., $\beta(u)=u^{p}$ for some $\left.p>0\right)$.

Hypothesis 2. The functionals $\psi$ and $F$ on $\mathscr{C}$ are continuous and $\psi$ is bounded. For all $Y \in \mathscr{C}, F$ satisfies

$$
F(Y) \geqq-b\|Y\|_{2}^{2}-c_{2}(\varepsilon)-\varepsilon\left\|A^{-\Delta} Y\right\|_{2}^{2}
$$

for some $0 \leqq b<1 /\left(2\|A\|_{2}\right)$, some $0 \leqq \Delta<1 / 2$ such that $\operatorname{Tr}\left(A^{1-2 \Delta}\right)<\infty$, and all sufficiently small $\varepsilon>0$.

Under Hypothesis 2, $G$ has minimum points [Simon (2); Lemma 18.5]. We denote by $M$ the set of minimum points of $G$.

Hypothesis 3. The functionals $\psi$ and $F$ are smooth on some neighborhood $\mathscr{V}$ of $M$. For $\bar{Y} \in \mathscr{V}, Y_{1}, Y_{2} \in \mathscr{C}$, the first and second Frechet derivatives of $F$ at $\bar{Y}$ can be represented as

$$
D F(\bar{Y}) Y_{1}=\left\langle F^{\prime}(\bar{Y}), Y_{1}\right\rangle, D^{2} F(\bar{Y})\left(Y_{1}, Y_{2}\right)=\left\langle F^{\prime \prime}(\bar{Y}) Y_{1}, Y_{2}\right\rangle,
$$

where $F^{\prime}(\bar{Y})$ is a smooth $\mathscr{H}$-valued function and $F^{\prime \prime}(\bar{Y})$ is a smooth $\mathscr{B}(\mathscr{H})$-valued function.

Hypothesis 4. $M$ is a smooth $m$-dimensional nondegenerate submanifold of $\mathscr{C}$.

The next theorem, Theorem 5 , is the $\mathscr{b}$-analogue of Theorem 1 . All quantities in Theorem 5 are defined as in Theorem 1. (Note: for $x \in M, N_{x}=M_{x}^{\perp}$, where $\perp$ denotes orthogonal complement in $\mathscr{H}$.) The meaning of the asymptotic expansion in Theorem 5 is explained after Theorem 1 (with $\mathscr{H}$ replaced by $\mathscr{C}$ ).

Theorem 5. Let $P_{A}$ be a mean zero Gaussian probability measure on $\mathscr{C}$ with covariance operator $A$ and covariance function $A(s, t)$ and let $\psi, F$ be functionals on $\mathscr{C}$. Suppose that Hypotheses 1-4 are valid. Define the integrals $\left\{J_{n}\right\}$ by (1.1). Then

$$
\begin{aligned}
e^{n G^{*}} J_{n} \sim n^{m / 2} \int & \left\{\int_{N_{x}} \psi(x+z / \sqrt{n}) I_{M}(x, z / \sqrt{n}) \exp \left(-n F_{3}(x, z / \sqrt{n})\right) d P_{B(x)}(z)\right\} . \\
& \cdot c(x) d V_{M}(x) .
\end{aligned}
$$


To prove Theorem 5, we must make suitable modifications in the proofs of Lemmas 1, 2, 3, 7 and of Theorem 1. Concerning the other lemmas, in Lemma 4 we change $\mathscr{H}$ to $\mathscr{C}$ and $K^{\delta}$ to $K_{\delta}$, where

$$
K^{\delta} \doteq\left\{Y \mid \inf _{x \in K}\|Y-x\|_{2}<\delta\right\}, K_{\delta} \doteq\left\{Y \mid \inf _{x \in K}\|Y-x\|<\delta\right\} .
$$

The proof of Theorem 5 uses this new Lemma 4, the proof of which is simple, together with Lemmas 5,6, and 8 as stated, the proofs of which stay unchanged. (The conclusions of Lemma 8 stay valid since continuity in $\mathscr{C}$ implies continuity in $\mathscr{H}$.) We next modify Lemmas 1, 2, 3,7 and the proof of Theorem 1 .

Lemma 1 holds as stated. The only fact that requires separate proof is (a), namely that $M$ is a compact subset of $\mathscr{C}$ (and thus of $\mathscr{H}$ ). This follows from the proof of [Ellis-Rosen (2); Lemma 4.5 (d)] and the fact that if $F$ satisfies (6.2), then there exist $b>0, \varepsilon>0$ such that for all $Y \in \mathscr{C} G(Y) \geqq-c_{2}(\varepsilon)+b I(Y)$.

Lemma 2 holds as stated. For use below (Lemmas 3 and 7) we augment Lemma 2 to show the following technical result on the tightness of certain Gaussian measures.

Lemma 9. Suppose that $A$ satisfies Hypothesis 1. Let $\Gamma$ be an index set and $\left\{V_{\gamma} ; \gamma \in \Gamma\right\}$ finite dimensional subsets in $\mathscr{D}\left(A^{-1}\right)$ all of the same dimension $d$. Suppose that for each $\gamma \in \Gamma$ we have a bounded linear operator $L_{\gamma}$ such that for all $v \in V_{\gamma}$

and suppose that

$$
v=A L_{\gamma} v
$$

$$
\sup \left\{\left\|L_{\gamma}\right\|_{2},\left\|\left(A_{V_{\gamma}}\right)^{-1}\right\|_{2} \mid \gamma \in \Gamma\right\} \leqq c^{\prime}<\infty .
$$

Then the set of Gaussian measures $\left\{P_{V_{\gamma}^{\frac{1}{\gamma}}} ; \gamma \in \Gamma\right\}$ is tight.

Proof. By Theorem $A$ in the Appendix of this paper, it suffices to prove that the covariance functions of the $\left\{P_{V_{\nu}^{\perp}} ; \gamma \in \Gamma\right\}$ all satisfy (6.1) for some $c, \beta$ independent of $\gamma \in \Gamma$. For each $\gamma$, let $\left\{\phi_{\gamma, i} ; i=1, \ldots, d\right\}$ be an orthonormal basis of $V_{\gamma}$. Denoting the orthogonal projections $\pi_{V_{\gamma}}$ and $\pi_{V_{\nu}^{\perp}}$ by $\pi_{\gamma}$ and $\pi_{\gamma}^{\perp}$, respectively, we have $\pi_{\gamma}^{\perp}=$ $I-\pi_{\gamma}$ and for $Y \in \mathscr{H}$

$$
\left(\pi_{\gamma} Y\right)(s)=\int_{0}^{1} \sum_{i=1}^{d} \phi_{\gamma, i}(s) \phi_{\gamma, i}(t) Y(t) d t, 0 \leqq s \leqq 1 .
$$

Now (2.15)implies

Since by $(6.5)$

$$
A^{V_{\nu}^{\perp}}=\pi_{\gamma}^{\perp} A \pi_{\gamma}^{\perp}-\pi_{\gamma}^{\perp} A \pi_{\gamma}\left(\pi_{\gamma} A \pi_{\gamma}\right)^{-1} \pi_{\gamma} A \pi_{\gamma}^{\perp} .
$$

$$
\sup _{\gamma \in \Gamma}\left\{\left\|\left(\pi_{\gamma} A \pi_{\gamma}\right)^{-1}\right\|_{2}\right\} \doteq \sup _{\gamma \in \Gamma}\left\{\left\|\left(A_{V_{\gamma}}\right)^{-1}\right\|_{2}\right\} \leqq c^{\prime}<\infty,
$$

we are done once we show the following: for all $\gamma \in \Gamma, i \in\{1, \ldots, d\}$

$$
\left|\phi_{\gamma, i}(s)\right| \leqq \bar{c}<\infty\left|\phi_{\gamma, i}(s)-\phi_{\gamma, i}(t)\right| \leqq \bar{\beta}\left(\left|s-s^{\prime}\right|\right), 0 \leqq s, s^{\prime}, t \leqq 1,
$$

where $\bar{\beta}$ is some function as in Hypothesis 1 . Since $A(s, t)$ satisfies (6.1), we have by (6.4)

$\left|\phi_{\gamma, i}(s)\right| \leqq \sup \{|A(s, t)| \mid 0 \leqq s, t \leqq 1\}\left\|L_{\gamma}\right\|_{2} \leqq c c^{\prime}<\infty, \gamma \in \Gamma, i \in\{1, \ldots, d\}, s \in[0,1]$. 
This gives the first bound in (6.7). The second bound in (6.7) follows similarly from the second bound in (6.1).

We now modify the proof of Theorem 1 . In Lemma 3, we assume that $A$ also satisfies Hypothesis 1 and that $K$ is a compact, finite dimensional submanifold of $\mathscr{C}$. We still use $K^{\delta}$, the $\mathscr{H}$-neighborhood of $K$ which forms a uniformly tubular neighborhood, but we assume that $\phi$ is a bounded, continuous functional on $\mathscr{C}$. These hypotheses on $\phi$ differ from the hypotheses on $\phi$ made in the original version of Lemma 3 ( $\phi$ bounded and uniformly continuous). The technical reasons for these new hypotheses will be explained below, just before the statement of Lemma 10. We note that Lemma 3 applies to the choice $K=\sqrt{n} M$ (see discussion after Lemma 3). Indeed, since by Lemma $1(b) A^{-1} x=F^{\prime}(x)$ for $x \in M$, Hypothesis 6.4 implies that $A^{-1} x$ is a smooth $\mathscr{H}$-valued function of $x$ on $\sqrt{n} M$. We prove this modified Lemma 3 later.

The crucial point in our modification of the proof of Theorem 1 lies in the choice of the cutoff function $f$. On the one hand, we need a uniformly tubular neighborhood for Lemma 3- and our tubular neighborhoods are $\mathscr{H}$ objects-while on the other hand, in order to expand $\psi$ and $F$ we need neighborhoods in $\mathscr{C}$. We now choose $f$. Pick $\delta$ so small that $M^{(2 \delta)}$ is a uniformly tubular neighborhood of $M$ and define the following subsets of $M^{(2 \delta)}$ :

$$
\begin{aligned}
& S_{1}(\delta) \doteq\left\{Y \mid Y=x+z, x \in M, z \in N_{x},\|z\| \leqq \delta\right\}, \\
& S_{2}(\delta) \doteq\left\{Y \mid Y=x+z, x \in M, z \in N_{x},\|z\| \geqq \delta,\|z\|_{2} \leqq \delta\right\} .
\end{aligned}
$$

We will show below that $S_{1}(\delta)$ and $S_{2}(\delta)$ are closed in $\mathscr{C}$. Since $\left(M^{\delta}\right)^{c}$ is closed in $\mathscr{C}$, Urysohn's lemma [Royden; Prop. 8.2] guarantees the existence of a continuous functional $f$ on $\mathscr{C}$ which satisfies $f=1$ on $S_{1}(\delta / 2), f=0$ on $S_{2}(\delta) \cup\left(M^{\delta}\right)^{c}$, and $0 \leqq f \leqq 1$.

We next prove that

$$
\begin{aligned}
e^{n G^{*}} J_{n} & \doteq e^{n G^{*}} \int \psi(Y / \sqrt{n}) \exp (-n F(Y / \sqrt{n})) d P_{A}(Y) \\
& =e^{n G^{*}} \int f(Y / \sqrt{n}) \psi(Y / \sqrt{n}) \exp (-n F(Y / \sqrt{n})) d P_{A}(Y)+O\left(e^{-n c}\right)
\end{aligned}
$$

for some $c=c(\delta)>0$. Since $f=1$ on the open neighborhood $\left[S_{2}(\delta / 2) \cup(M \delta / 2)^{c}\right]^{c} \subseteq$ $S_{1}(\delta / 2)$ of $M,(6.8)$ follows once we have shown

$$
e^{n G^{*}} \int_{\mathscr{L}} e^{-n F(Y)} d P_{A}(\sqrt{n} Y)=O\left(e^{-n c}\right)
$$

where $\mathscr{L}$ is any closed subset of $\mathscr{C}$ such that $\mathscr{L} \cap M=\phi$ and $c=c(\mathscr{L})>0$. To prove (6.9), we verify

$$
\lim _{L \rightarrow \infty} \lim _{n \rightarrow \infty} \sup (1 / n) \log \int_{\{Y:-F(Y) \geqq L\}} e^{-n F(Y)} d P_{A}(\sqrt{n} Y)=-\infty,
$$

then appeal to [Varadhan; Sect. 3] and the $\mathscr{C}$-analogue of [Ellis-Rosen (1); Lemma 4.1] (the proof of the latter is easily adapted to the case of $\mathscr{C}$ ). The limit (6.10) follows from the bound (6.2) on $F$ and the fact that

$$
\int \exp \left[\varepsilon\left\langle Y, A^{-2 \Delta} Y\right\rangle\right] d P_{A}(Y)<\infty
$$


for all sufficiently small $\varepsilon>0$ [Simon (2); Lemma 18.2]. This proves (6.8).

We continue with our modification of the proof of Theorem 1, working with the second integral in (6.8). The functionals $\psi$ and $F$ are assumed to be smooth on some neighborhood $\mathscr{V}$ of $M . \mathscr{V}$ contains $M_{\bar{\delta}}$ for some $\bar{\delta}>0$. Choosing $\delta<\bar{\delta}$, we have

$$
\operatorname{supp} f \subseteq M_{\bar{\delta}} .
$$

Thus, $\psi$ and $F$ are smooth on the support of $f$. For sufficiently small $\delta$, we can expand $\psi(x+z / \sqrt{n})$ and $F_{3}(x+z / \sqrt{n})$ in the normal direction $z$ since we have $\|z\| / \sqrt{n}<\delta$ in (3.7). Finally, since $f=1$ on $S_{1}(\delta / 2)$, the proof of Theorem 5 can be completed as in Sect. 3 once we prove the $\mathscr{C}$-analogue of Lemma $7\left(\|-\|_{2}\right.$ replaced by $\|-\|$ ).

We next prove that $S_{1}(\delta)$ and $S_{2}(\delta)$ are closed in $\mathscr{C}$, then prove the modified versions of Lemmas 3 and 7.

To show the closure of $S_{1}(\delta)$, let $\left\{Y_{i}\right\}$ be a sequence in $S_{1}(\delta)$ such that $Y_{i} \rightarrow Y$, where $Y$ is some element in $\mathscr{C}$. For each $i$, we write $Y_{i}=x_{i}+z_{i}$, where $x_{i} \in M$, $z_{i} \in N_{x_{i}}$. By the compactness of $M$, there exists a subsequence $\left\{i_{1}\right\}$ of $\{i\}$ and an element $x \in M$ such that $x_{i_{1}} \rightarrow x$. Then $z_{i_{1}}=Y_{i_{1}}-x_{i_{1}} \rightarrow Y-x$. Define $z \doteq Y-x$. Since clearly $\|z\| \leqq \delta / 2$, we need only show $z \in N_{x}$. Since $M$ is a smooth manifold, for each $w$ near $x$ we can find a basis $\left\{\phi_{j, w} ; j=1,2, \ldots, m\right\}$ for $M_{w}$, with each $\phi_{j, w}$ smooth in $w$. For each $j \in\{1, \ldots, m\}$, we have as $i_{1} \rightarrow \infty$

$$
\left\langle z_{i_{1}}, \phi_{j, x_{i_{1}}}\right\rangle \rightarrow\left\langle z, \phi_{j, x}\right\rangle \text {. }
$$

But $\left\langle z_{i_{1}}, \phi_{j, x_{i_{1}}}\right\rangle=0$ since $z_{i_{1}} \in N_{x_{i_{1}}}$, and so we conclude that $z \in N_{x}$. This proves the closure of $S_{1}(\delta)$. A similar proof yields the closure of $S_{2}(\delta)$.

We now prove the modified version of Lemma 3 ( $K$ a compact, finite dimensional submanifold of $\mathscr{C}$ and $\phi$ a bounded, continuous functional on $\mathscr{C}$ ). We find that we must modify the proofs of Assertions (a) and (b). As in Sect. 3, Lemma 3 is applied to the functional (see paragraph after (3.5))

$$
\phi(Y)=f(Y / \sqrt{n}) \psi(Y / \sqrt{n}) \exp (-n F(Y / \sqrt{n}))
$$

Because of the choice of the cutoff functional $f$, this $\phi$ was uniformly continuous in Sect. 3 while in the present section it is only continuous. This explains the new hypotheses for Lemma 3. The uniform continuity of $\phi$ allowed us to prove the original version of Assertion (a) in a relatively simple way. We now show that the boundedness and continuity of $\phi$ suffice. To prove the modified version of Assertion (a), we need a lemma, which will also be used in the proof of Assertion (b).

Lemma 10. The set of measures $\left\{P_{N_{x}} ; x \in K\right\}$ is tight. Also, there exists $j_{0}$ such that the set of measures $\left\{P_{N_{j}, \pi_{j x}} ; j \geqq j_{0}, x \in K\right\}$ is tight.

Proof. We first prove the tightness of $\left\{P_{N_{x}} ; x \in K\right\}$. By hypothesis in Lemma 3, $A^{-1} x$ is a smooth $\mathscr{H}$-valued function of $x$ on $K$, say $H(x)$. Then for $x \in K$

so that for $v \in K_{x}$

$$
x=A H(x),
$$

$$
v=A H^{\prime}(x) v .
$$


We now appeal to Lemma 9 with $\Gamma \doteq K$ and $V_{\gamma} \doteq K_{x}$ for $\gamma=x \in K$. The hypotheses of Lemma 9 are verified using the smoothness of $H^{\prime}(x)$ and (4.22). We conclude that $\left\{P_{N_{x}} ; x \in K\right\}$ is tight.

We now prove the tightness of $\left\{P_{N_{j, \pi_{j} x}} ; j \geqq j_{0}, x \in K\right\}$ for some sufficiently large $j_{0}$. We choose $j_{0}$ so large that for all $j \geqq j_{0}, \pi_{j}$ is an embedding of $K$ onto $\pi_{j} K$. In particular, $\pi_{j}$ is an isomorphism of $K_{x}$ onto $K_{j, \pi_{j} x}$ which we denote by $\tilde{\pi}_{j, x^{*}}$ By (6.12), we have for $v \in K_{x}$

$$
\pi_{j} v=A \pi_{j} H^{\prime}(x) v=A\left(\pi_{j} H^{\prime}(x) \tilde{\pi}_{j, x}^{-1}\right) \pi_{j} v
$$

i.e., for $u \in K_{j, \pi_{j} x}$

$$
u=A\left(\pi_{j} H^{\prime}(x) \tilde{\pi}_{j, x}^{-1}\right) u .
$$

Using (4.18), we can then verify the hypotheses of Lemma 9 with $\Gamma \doteq K \times\left\{j \mid j \geqq j_{0}\right\}$, $V_{\gamma} \doteq K_{j, \pi_{j} x}$ for $\gamma=(x, j)$. We conclude that $\left\{P_{N_{j, \pi_{j} x}} ; j \geqq j_{0}, x \in K\right\}$ is tight.

We now prove the modified version of Assertion (a). By Lemma 10, there exists $j_{0}$ such that the set of measures in (4.28) in the $z$-variable (i.e., the measures $\left.\left(P_{N_{j, \pi_{j} x}} ; j \geqq j_{0}, x \in K\right\}\right)$ is tight. Since the integrands are bounded, we may restrict our attention to those $z$ lying in some compact set $S$. It suffices to prove that for any $\varepsilon>0$ we have

$$
\sup _{x \in K, z \in S}\left|\phi\left(\pi_{j} x+z\right)-\phi(x+z)\right|<\varepsilon
$$

for all sufficiently large $j$. By (6.1),

$$
\|A Y\| \leqq \text { const }\|Y\|_{2}
$$

for all $Y \in \mathscr{H}$. By (6.11)

$$
\left\|\pi_{j} x-x\right\|=\left\|A\left(\pi_{j} H(x)-H(x)\right)\right\| \leqq \text { const }\left\|\pi_{j} H(x)-H(x)\right\|_{2} .
$$

Since $H(x)$ is continuous and $K$ is compact, we see that $\pi_{j} x \rightarrow x$ uniformly on $K$. The inequality (6.15) now follows from the continuity of $\phi$ and the compactness of $K \times S$. This completes the proof of the modified version of Assertion (a).

In Assertion (b), we need to prove that for each $x \in K$

$$
P_{N_{j, \pi_{j} x}} \rightarrow P_{N_{x}} \text { weakly on } \mathscr{C} \text {. }
$$

We denote by $A^{N_{j, \pi_{j} x}}(s, t)$ and $A^{N_{x}}(s, t)$ the covariance functions of $P_{N_{j, \pi_{j} x}}$ and $P_{N_{x}}$, respectively. We next prove

$$
A^{N_{j, \pi_{j} x}}(s, t) \rightarrow A^{N_{x}}(s, t), \quad 0 \leqq s, t \leqq 1, \quad \text { as } j \rightarrow \infty .
$$

The weak convergence in (6.16) will follow from (6.17) and the tightness of the measures $\left\{\boldsymbol{P}_{N_{j, \pi_{j} x}} ; j \geqq j_{0}\right\}$, proved in Lemma 10. Concerning (6.17), let $\left\{\boldsymbol{\phi}_{i, x} ; i=\right.$ $\left.1, \ldots, k \doteq \operatorname{dim}\left(K_{x}\right)\right\}$ be an orthonormal basis of $K_{x}$. Then for all $j$ sufficiently large, the elements $\left\{\pi_{j} \phi_{i, x} ; i=1, \ldots, k\right\}$ are linearly independent, and so an orthonormal basis of $K_{j, \pi_{j} x}$ can be constructed from them. We claim that

$$
\pi_{j} \phi_{i, x} \rightarrow \phi_{i, x} \text { in } \mathscr{C}, \quad i=1, \ldots, k \text {. }
$$

Then (6.17) follows from (6.18) and the representations (6.5) and (6.6) with 
$A \doteq A^{N_{j, \pi_{j} x}}$ and $A \doteq A^{N_{x}}$. Using (6.12) and (6.16), we have for $v \in K_{x}$

$$
\left\|v-\pi_{j} v\right\|=\left\|A\left(H^{\prime}(x) v-\pi_{j} H^{\prime}(x) v\right)\right\| \leqq \text { const }\left\|H^{\prime}(x) v-\pi_{j} H^{\prime}(x) v\right\|_{2},
$$

which tends to 0 as $j \rightarrow \infty$. This proves (6.18) and completes the proof of the modified version of Assertion (b).

We now prove the modified version of Lemma 7. It suffices to show the existence of $b>0, d<\infty$ such that for all $a>0$ and $x \in M$

$$
P_{B(x)}\{z \mid\|z\| \geqq a\} \leqq d e^{-b a^{2} / 2} .
$$

Let $Q$ be any mean zero Gaussian measure on $\mathscr{C}$. The proof of Theorem 1.9 in [Marcus-Shepp] shows that if for some $s>0$,

$$
Q\{Y \mid\|Y\| \leqq s\} \geqq q>1 / 2,
$$

then for all $a>s$

$$
Q\{Y \mid\|Y\|>a\} \leqq e^{-t a^{2}},
$$

where $t \doteq\left(24 s^{2}\right)^{-1} \log [q /(1-q)]$. We prove (6.19) by finding $s>0$ and $q>1 / 2$ such that for all $x \in M$

$$
P_{B(x)}\{z \mid\|z\| \leqq s\} \geqq q .
$$

To prove (6.20), it suffices to find a compact subset $\mathscr{T}$ of $\mathscr{C}$ such that

$$
P_{B(x)}(\mathscr{T}) \geqq 3 / 4 \text { for all } x \in M \text {. }
$$

By Lemma 10 , for any $\varepsilon \in(0,1)$, we can find a compact subset $\mathscr{T}_{\varepsilon} \subset \mathscr{C}$ such that

$$
P_{N_{x}}\left(\mathscr{T}_{\varepsilon}\right) \geqq 1-\varepsilon \text { for all } x \in M \text {. }
$$

We now use [Ellis-Rosen (1); Lemma 4.4] with $\mathscr{H}_{1} \doteq N_{x}, A_{1} \doteq A^{N_{x}}, \Lambda \doteq\left(F^{\prime \prime}(x)\right)_{N_{x^{\prime}}}$ and $B_{1} \doteq B(x)$, for $x \in M$ (cf., (4.30) above). We have $P_{B(x)} \ll P_{N_{x}}$ and as one can show,

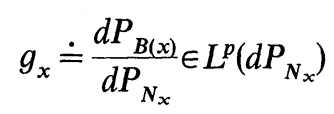

for some $p>1$. In fact, using (4.23)(a) above, we can choose $p>1$ so that

$$
\left\|g_{x}\right\|_{p}=\left[\int\left|g_{x}\right|^{p} d P_{N_{x}}\right]^{1 / p} \leqq \gamma<\infty \text { for all } x \in M
$$

By (6.22)-(6.24), we have for any $\varepsilon \in(0,1)$

$$
P_{B(x)}\left\{\mathscr{T}_{\varepsilon}^{c}\right\} \leqq \gamma\left[P_{N_{x}}\left\{\mathscr{T}_{\varepsilon}^{c}\right\}\right]^{1 / q}<\gamma \varepsilon^{1 / q},
$$

where $q$ is the conjugate index to $p$. Now (6.21) follows if we choose $\varepsilon$ sufficiently small. This proves the modified version of Lemma 7 and completes the proof of Theorem 5. 


\section{Appendix}

We prove a result on the tightness of certain Gaussian measures on $C[0,1]$. This result is used in the proof of Lemma 9 in Sect. 6.

Theorem A1. Let $\Gamma$ be an index set and $\left\{P_{\gamma} ; \gamma \in \Gamma\right\}$ a family of mean zero Gaussian measures on $C[0,1]$ with covariance functions $\left\{A_{\gamma} ; \gamma \in \Gamma\right\}$. Define $\zeta(u) \doteq u^{-1}$. $[\log (1 / u)]^{-1 / 2}$. Assume that there exist numbers $c \in(0, \infty)$ and $\varepsilon \in(0,1 / e)$ and a nondecreasing function $\beta$ on $[0,1]$ which is continuous on $[0, \varepsilon]$ and satisfies $\beta(0)=0$. Assume that

$$
\begin{gathered}
A_{\gamma}(0,0) \leqq c \text { for each } \gamma \in \Gamma, \\
\left|A_{\gamma}(s, t)-A_{\gamma}\left(s^{\prime}, t\right)\right| \leqq \\
\beta\left(\left|s-s^{\prime}\right|\right) \text { for each } \gamma \in \Gamma, \text { all } 0 \leqq s, s^{\prime}, t \leqq 1, \\
\int_{0}^{\varepsilon} \beta(u) \zeta(u) d u<\infty .
\end{gathered}
$$

Then the family $\left\{\boldsymbol{P}_{\gamma} ; \gamma \in \Gamma\right\}$ is tight.

Proof. By [Billingsley; Theorem 8.2], we must prove that for each sequence $\left\{P_{n} ; n=1,2, \ldots\right\} \subseteq\left\{P_{\gamma} ; \gamma \in \Gamma\right\}$ and for each $\varepsilon>0$, there exists an $a \geqq 0$ such that

$$
P_{n}\{Y|| Y(0) \mid>a\} \leqq \varepsilon, \text { each } n
$$

We must also prove that for each $\alpha>0, \varepsilon>0$, there exists a $\delta \in(0,1)$ and an integer $n_{0}$ such that

$$
P_{n}\left\{Y\left|\sup _{|s-t| \leqq \delta}\right| Y(s)-Y(t) \mid \geqq \alpha\right\} \leqq \varepsilon, n \geqq n_{0}
$$

The bound (A.4) holds because of (A.1). By a straightforward extension of the proof of [Jain-Marcus; Theorem IV. 1.3] one proves that there exists numbers $s_{m} \rightarrow 0$ as $m \rightarrow \infty$ such that for all $m$ sufficiently large, all $h$ satisfying $2^{-m-1}<h \leqq$ $2^{-m}$, and all $n$

$$
P_{n}\left\{Y\left|\max _{j=1, \ldots, 2^{m}} \sup _{|u| \leqq h}\right| Y\left(j 2^{-m}+u\right)-Y\left(j 2^{-m}\right) \mid \geqq \alpha(h)\right\} \leqq s_{m},
$$

where $\alpha(h) \doteq$ const $\left[\int_{0}^{h} \beta(u) \zeta(u) d u+(\log 1 / h)^{1 / 2} \beta(h)\right]$. Since $\alpha(h) \rightarrow 0$ as $h \rightarrow 0,($ A.5)
follows.

\section{References}

Billingsley, P. : Convergence of Probability Measures. New York: John Wiley and Sons 1968

Coleman, S. : The Uses of Instantons. Lectures delivered at the 1977 International School of Subnuclear Physics, Ettore Majorana

Donsker, M. D., Varadhan, S. R. S. : Commun. Pure Appl. Math. 29, 389-461 (1976)

Ellis, R. S., Rosen, Jay S. : 1. Asymptotic analysis of Gaussian integrals, I. : isolated minimum points. Trans. Am. Math. Soc. (to appear). 2. Laplace's method for Gaussian integrals with an application to statistical mechanics. Annals of Probab. (to appear)

Gihman, I. I., Skorohod, A. V.: The Theory of Stochastic Processes I. Transl. by S. Kotz, Berlin, Heidelberg New York. : Springer 1974

Guillemin, V., Pollack, A. : Differential topology. Englewood Cliffs, N. J. : Prentice Hall, 1974

Jain, N. C., Marcus, M. B. : Continuity of subgaussian processes. Univ. of Minn./Northwestern Univ. Preprint (1978) 
Marcus, M. B., Shepp, L. A. : Sample behavior of Gaussian processes. In : Sixth Berkeley Symposium on Math. Stat. and Probability, Vol. II, 423-439 (1972)

Pincus, M. : Trans. Am. Math. Soc. 134, 193-216(1968)

Royden, H. L. : Real analysis. New York: Macmillan 1963

Schilder, M. : Trans. Am. Math. Soc. 125, 63-85 (1966)

Simon, B.: 1. Functional Integration and Quantum Physics. New York: Academic Press 1979. 2. Trace Ideals and Their Applications. Cambridge: Cambridge Univ. Press 1979

Spivak, M. : A Comprehensive Introduction to Differential Topology. Vol. I-IV. Berkeley: Publish or Perish 1970

Varadhan, S. R. S. : Commun. Pure Appl. Math. 19, 261-286(1966)

Weyl, H. : Am. J. Math. 61, 461-472(1939)

Wiegel, F. W. : Phys. Rep. 16, 57-114 (1975)

Communicated by B. Simon

Received March 11, 1981 
